\title{
NASA Structural Analysis Report on the American Airlines Flight 587 Accident- Local Analysis of the Right Rear Lug
}

\author{
I. S. Raju*, E. H. Glaessgen ${ }^{\dagger}$, B. H. Mason ${ }^{\dagger}$, T. Krishnamurthy ${ }^{\dagger}$, and C. G. Dávila ${ }^{\dagger}$ \\ NASA Langley Research Center, Hampton, Virginia, 23681
}

\begin{abstract}
A detailed finite element analysis of the right rear lug of the American Airlines Flight 587 - Airbus A300-600R was performed as part of the National Transportation Safety Board's failure investigation of the accident that occurred on November 12, 2001. The loads experienced by the right rear lug are evaluated using global models of the vertical tail, local models near the right rear lug, and a global-local analysis procedure. The right rear lug was analyzed using two modeling approaches. In the first approach, solid-shell type modeling is used, and in the second approach, layered-shell type modeling is used. The solid-shell and the layered-shell modeling approaches were used in progressive failure analyses (PFA) to determine the load, mode, and location of failure in the right rear lug under loading representative of an Airbus certification test conducted in 1985 (the 1985-certification test). Both analyses were in excellent agreement with each other on the predicted failure loads, failure mode, and location of failure. The solid-shell type modeling was then used to analyze both a subcomponent test conducted by Airbus in 2003 (the 2003-subcomponent test) and the accident condition. Excellent agreement was observed between the analyses and the observed failures in both cases. From the analyses conducted and presented in this paper, the following conclusions were drawn. The moment, $M_{x}$ (moment about the fuselage longitudinal axis), has significant effect on the failure load of the lugs. Higher absolute values of $M_{x}$ give lower failure loads. The predicted load, mode, and location of the failure of the 1985-certification test, 2003-subcomponent test, and the accident condition are in very good agreement. This agreement suggests that the 1985-certification and 2003subcomponent tests represent the accident condition accurately. The failure mode of the right rear lug for the 1985-certification test, 2003-subcomponent test, and the accident load case is identified as a cleavage-type failure. For the accident case, the predicted failure load for the right rear lug from the PFA is greater than 1.98 times the limit load of the lugs.
\end{abstract}

\section{Introduction}

$\mathrm{O}$ N November 12, 2001, American Airlines Flight 587 (AA 587) crashed shortly after take-off killing all 260 people on board and 5 on the ground. The composite vertical tail of the aircraft separated from the fuselage resulting in loss of control and ultimately the loss of the aircraft.

Several teams at the NASA Langley Research Center were assembled to help the National Transportation Safety Board with this investigation. The internal NASA team was divided into several discipline teams including a structural analysis team that consisted of a global analysis team and a detailed lug analysis team. The global analysis team considered global deformations, load transfer, and failure modes within the composite vertical tail as well as failure of the composite rudder. The detailed lug analysis team focused on failure of the laminated composite lugs that attach the tail to the aluminum fuselage. This paper describes the analyses conducted by the detailed lug analysis team.

\footnotetext{
* Structures Discipline Expert, NASA Engineering and Safety Center, MS 155, AIAA Fellow.

${ }^{\dagger}$ Aerospace Engineer, Computational Structures and Materials Branch, MS 155, AIAA Senior Member.
} 
First, an overview of the problem, including the vertical tail plane (VTP) structure, is presented. Second, the various models developed for the right rear lug are described. Third, details of the material modeling, contact modeling, and progressive failure analysis (PFA) for solid-shell type modeling are presented. Fourth, a brief discussion of an alternative modeling approach, layered-shell modeling, is presented. Fifth, the global-local connection processes used to virtually embed the local lug model within a global model of the VTP are described. Sixth, the results of the analyses are presented. Finally, the results and lessons learned are discussed.

\section{Description of the Problem}

The vertical tail plane (VTP) of an Airbus A300-600R is connected to the fuselage with 6 lugs (3 on the righthand side and 3 on the left-hand side) through a pin and clevis connection (see Figures 1a to 1d). Six yokes (not shown in figures) also connect the VTP to the fuselage and take some of the lateral loads. The air loads on the VTP during the 12 seconds before the VTP separated from the fuselage were evaluated and were supplied to the NASA structures teams by the National Transportation Safety Board (NTSB) and Airbus. The air loads were derived from digital flight data recorder (DFDR) data obtained after the accident.

The NASA global analysis team and the Airbus team evaluated the loads on each of the lugs and determined that the right rear lug (see Figure 1d) carried the largest loads compared to the design allowable. The lug analysis team, therefore, focused on the detailed analysis of the right rear lug. The objectives of the lug analysis team were to predict the failure load, mode, and location in the right rear lug for the loading conditions that the right rear lug experienced during the accident.

The lug analysis team considered the right rear lug region shown in Figure 1d. The lug is a continuation of the skin of the vertical tail with two pre-cured fitting halves cured to either side of the skin in the vicinity of the lug hole (the fitting extends to rib 4, as shown in Figure 1d). The region modeled consists of the right rear lug, rib 1, the rear spar, and 6 stringers from rib 1 to rib 5. Two different modeling approaches were used. The first modeling approach involved the development of a finite element (FE) model of the region shown in Figure 1d using threedimensional (3D) elements in the region of the two pre-cured fitting halves of the lug and shell elements for the rest of the model and is termed the solid-shell model. The second modeling approach involved the development of an

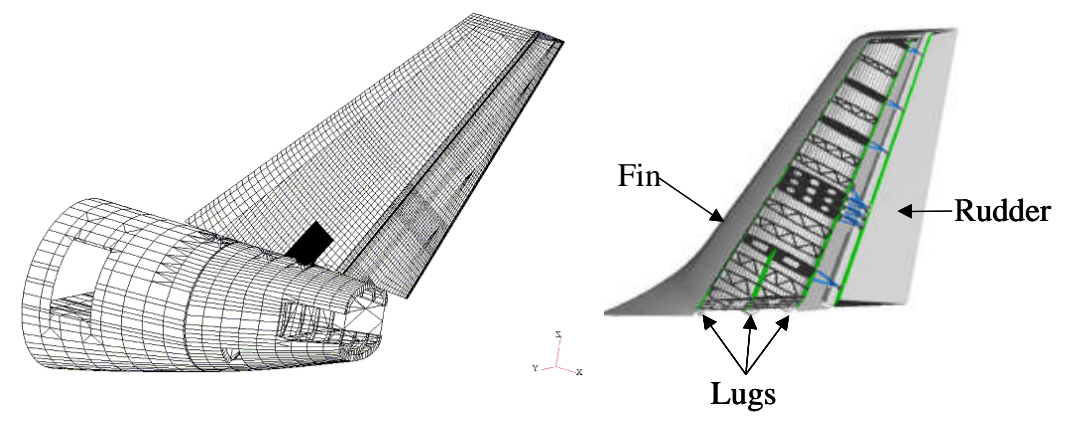

(a) A300-600R Empennage

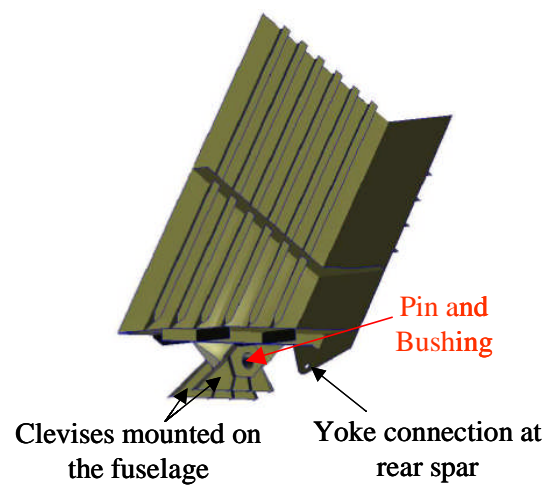

(c) VTP-Fuselage Connections (b) Major Components of Vertical Tail Plane (VTP)

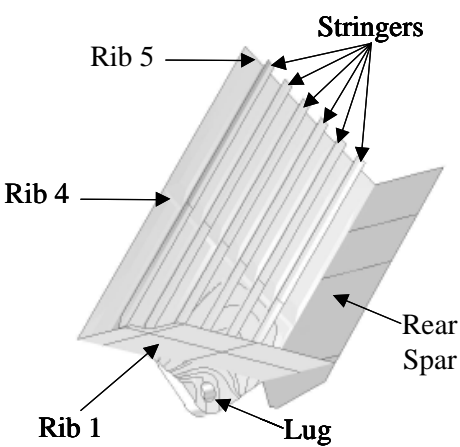

(d) Construction of Fin near Right Rear Lug

FE model of the region shown in Figure 1d using shell elements throughout and is termed the layeredshell model. In the layered-shell model, the 3D region of the first approach is modeled as shell layers that are connected by decohesion elements representing multi-point constraints. The results obtained by these approaches were validated by comparison with reference solutions for simplified configurations. The two approaches were also verified by comparing the finite element results with Airbus experimental results.

\section{Modeling}

The coarse 3D model (part of the solid-shell series of models) and layered-shell model were developed by modifying an Airbus-developed model of the same region. The damage modeling applied to each modeling approach was developed independently, which provided a degree of independent verification of the results from both methods. During the course of the investigation, two other solid-shell models were also

Figure 1. Vertical Tail Plane Mounted on the Fuselage. 


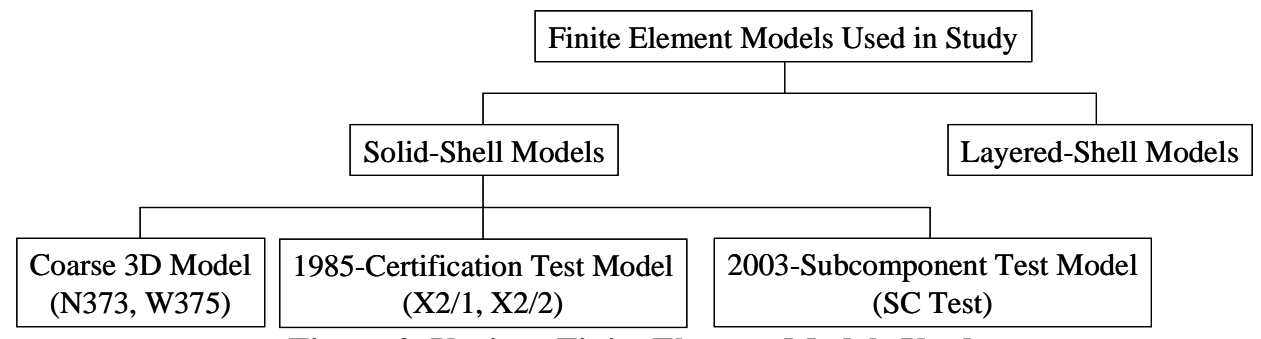

Figure 2. Various Finite Element Models Used.

developed. These models are the 1985certification test model and the 2003subcomponent (SC) test model. Figure 2 presents a summary of all the models used in the analyses - N373 and W375 denote different loading conditions; X2/1 and X2/2 represent two different specimens that were tested as part of an Airbus certification test conducted in 1985 (the 1985-certification test).

In the NASA-developed models, the clevises and the elastic pin with the bushing were not modeled. Rather, the pin is represented as a rigid analytical cylinder with a diameter equal to the diameter of the lug hole. This analytical cylinder is rigidly connected to an FE node at the location of the center of the pin. In the models, the pin is loaded by applying displacements or tractions to this single node. The pin loads are assumed to be reacted in the contact region between the lug hole and the pin.

The solid-shell and layered-shell analyses were performed using the commercial finite element code, ABAQUS [ABAQUS, 2000]. The code was chosen because it allows the user to implement specialized elements and material constitutive relationships while taking advantage of the features of a general-purpose code.

The progressive failure algorithms used to predict failure within the solid-shell and layered-shell models were defined as user defined material (UMAT) and user field (USFLD) algorithms, respectively. In the implementation of the UMAT and USFLD routines, material properties are degraded to small but nonzero values either in a single step or in several steps in each damaged element. To maintain stability of the system of equations, the values cannot be degraded to zero-values. Further, although some specialized codes allow failed elements to be removed from a model (element extinction), this capability is not available in ABAQUS. In the present implementation, the small stiffness contributions that remain in the degraded elements after failure allow a very small amount of load transfer across the damaged region. Therefore, in the present implementation of failure, complete separation of the lug is not possible.

\section{A. Coarse 3D Model}

A coarse 3D model (part of the solid-shell series of models) of the lug was developed using thickness contours extracted from the reference Airbus model. The coarse 3D model, shown in Figure 3a, has 25931 nodes and 21519 elements. The axial $(x-)$ coordinate is along the fuselage axis and is directed toward the rear of the airplane. The $y$ axis is parallel to the axis of the pin in the lug hole, and the $z$-axis is normal to the $x$-and $y$-axes. The lug fittings and skin are modeled with up to 14 layers of solid (8-node hexahedral) elements with 10 layers of elements in the vicinity of the hole. The thickness of each of the layers of solid elements was adjusted in order to match the volume of the lug fittings in the Airbus model. All other regions of the model were converted to shell elements. Multi-point constraint (MPC) equations were used at the solid-to-shell transition locations to ensure compatible translations and rotations along the interface.

\section{B. Layered Shell Model}

A layered-shell model of the lug was constructed using the same thickness contours as the coarse 3D model. The pin assembly was modeled as a rigid surface with a diameter equal to that of the lug hole. Frictionless contact equations were prescribed between the edge of the layered-shell elements around the bolt hole and the rigid surface. A discussion of the approximations caused by using a rigid frictionless pin can be found in Camanho and Matthews [Camanho and Matthews, 1999]. The lug fittings were modeled with 14 layers of shell elements, which were connected with 3D decohesion elements [Dávila et al., 2001a]. All other regions of the model were modeled with a single layer of shell elements. In addition, the model is used for progressive failure analyses in which the matrix and fiber damage is simulated by degrading the material properties. The analyses used for modeling the progressive delamination and intra-ply damage were developed within ABAQUS with user defined element (UEL) and USFLD user-written subroutines, respectively. This model, shown in Figure 3b, has 20886 nodes with 34524 elements.

The ability of the coarse 3D and layered-shell models to predict the same displacements as the original Airbus model was verified. Both the magnitude and spatial distributions of the displacement components predicted by the two NASA models were in very close agreement with those predicted by the Airbus model. 


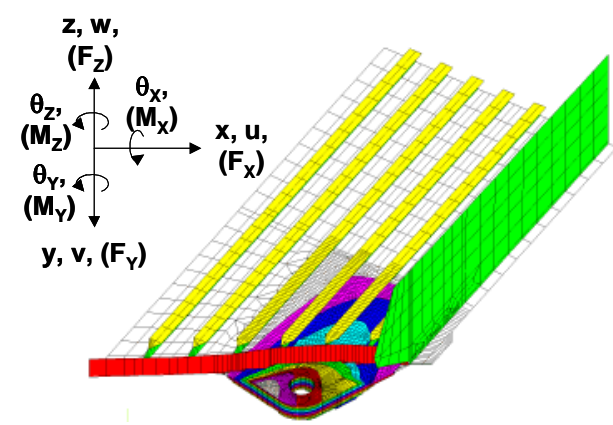

(a) Solid-Shell Model

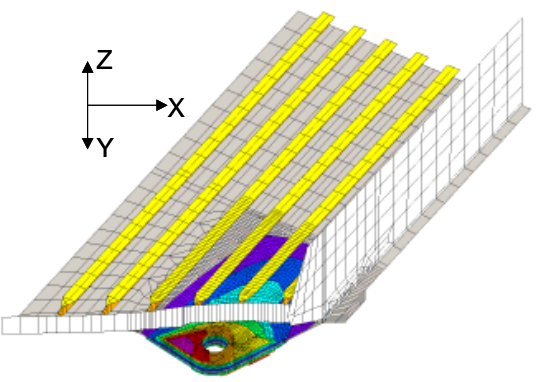

(b) Layered Shell Model

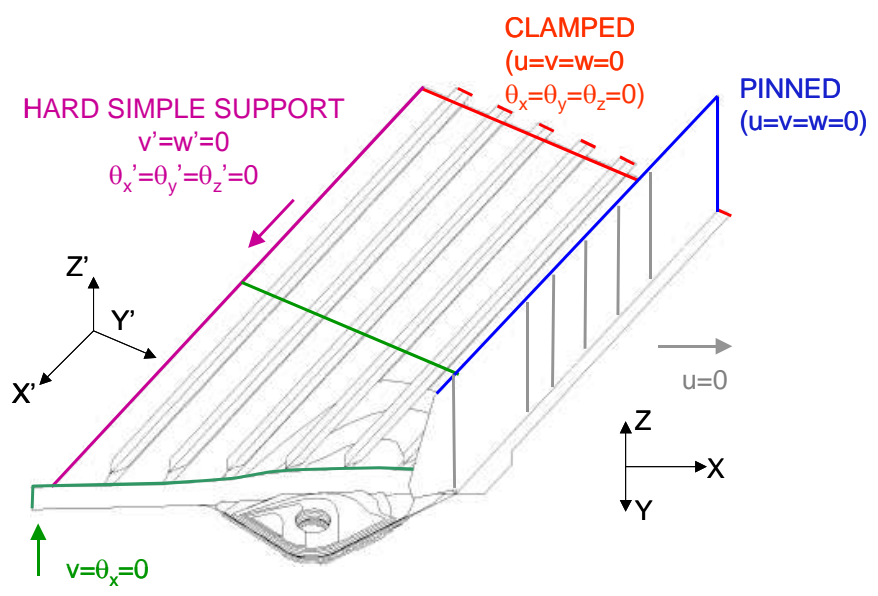

(c) Initial Airbus Boundary Conditions

Figure 3. Finite Element Models of Right Rear Lug (colors are for visualization purposes only).

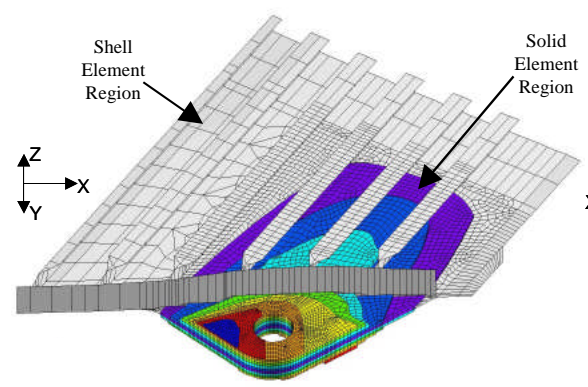

(a) Finite Element Model

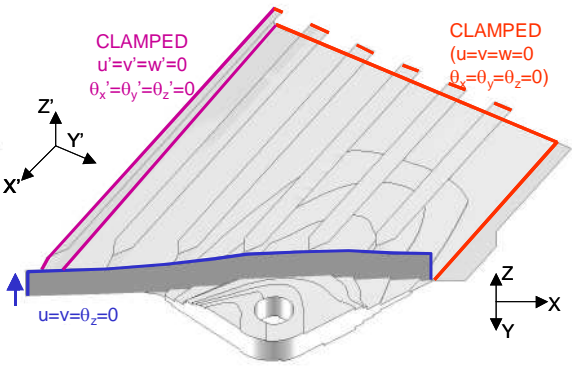

(b) Boundary Conditions

Figure 4. 1985-Certification Test Model of X2/2 Specimen (colors are for visualization purposes only).

\section{1985-Certification Test Model}

Two test specimens (called X2/2 and X2/1) were tested by Airbus in 1985. One FE model was used to represent both test specimens. To simulate the configurations of the X2/2 and X2/1 test specimens, an FE model was created from the coarse 3D model by deleting all the elements above rib 4 and forward of stringer 6 as shown in Figure 4a. This model had 23216 nodes and 19149 elements. The boundary conditions used with this model are shown in Figure 4b. 


\section{2003-Subcomponent Test Model}

As part of the investigation, a subcomponent test was conducted during 2003 on a left rear lug made of the same material as the accident aircraft. A left rear lug was used because this was the only rear lug (with the same material as the accident aircraft) that was available at the time of the test. Airbus modeled this left rear lug (see Figure 5a) including the support structure and supplied the model to the lug analysis team. This Airbus model then became part of the solid-shell series of models. The boundary conditions for this model are shown in Figure 5b. When this model is used to represent the right rear lug, the loads and boundary conditions are mirrored about the global $x z$ plane; i.e. the sign of $F_{Y}, M_{X}, M_{Z}, v, \theta_{X}$, and $\theta_{Z}$ are reversed.

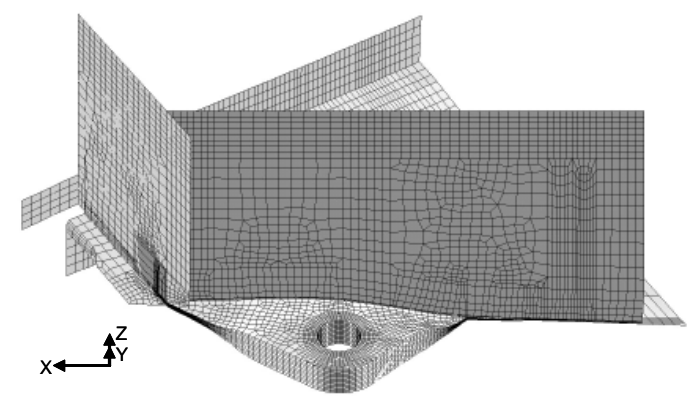

(a) Finite Element Model

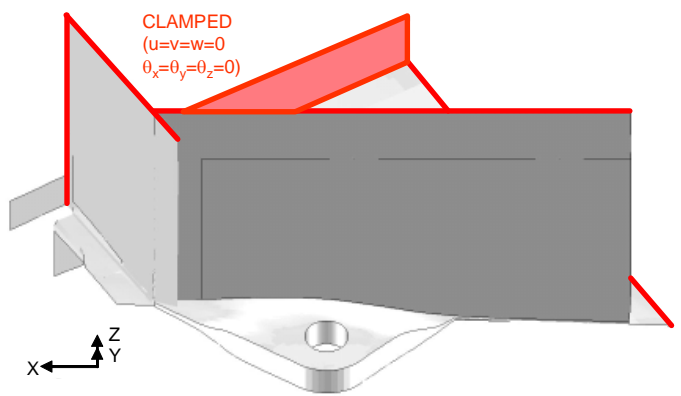

(b) Boundary Conditions (prescribed along the red edges and the red region)

Figure 5. 2003- Subcomponent Test Model.

\section{Solid Element Models}

\section{A. Material Modeling}

The right rear lug consists of two pre-cured fitting-halves, the vertical tail plane (VTP) skin and several compensation layers. The inner fitting-half, skin, and outer fitting-half are made from T300/913C in the form of $\pm 45^{\circ}$ fabric, $90^{\circ} / 0^{\circ}$ fabric, and $0^{\circ}$ tape and are approximately $55 \mathrm{~mm}$ thick in the vicinity of the pin.

Table 1 shows the elastic, strength, and toughness parameters for T300/913C from the recent World Wide Failure Exercise (WWFE, Soden and Hinton, 1998a and Soden and Hinton, 1998b). The subscripts 1, 2, and 3 denote the fiber direction, in-plane transverse direction, and out-of-plane direction, respectively, and the subscript "c" denotes a compressive property. Also, $X_{T}, X_{C}, Y_{T}$, and $Y_{C}$ denote the fiber-direction tensile strength, fiber-direction compressive strength, transverse-direction tensile strength, and transverse-direction compressive strength, respectively. Finally, $G_{I C}$ is the mode-I interlaminar fracture toughness.

\section{Homogenization of Material Properties}

The right rear lug contains numerous plies of T300/913C in the form of tape and fabric. Although a finite element model that explicitly modeled each of the plies and each of the numerous curvilinear ply drops within the lug could be developed, doing so would have required a finite element model with millions of elements. Such a detailed finite

Table 1. Material Properties for T300/913C Tape.

\begin{tabular}{|c|c|}
\hline Property & $\begin{array}{c}\text { WWFE } \\
\text { [Hinton and Soden 1998] }\end{array}$ \\
\hline$E_{1}(\mathrm{GPa})$ & 138 \\
\hline$E_{1 C}(\mathrm{GPa})$ & -- \\
\hline$E_{2}(\mathrm{GPa})$ & 11 \\
\hline$E_{2 C}(\mathrm{GPa})$ & -- \\
\hline$v_{12}$ & 0.28 \\
\hline$v_{23}$ & 0.4 \\
\hline$\mu_{12}(\mathrm{GPa})$ & 5.5 \\
\hline$X_{T}(\mathrm{MPa})$ & 1500 \\
\hline$X_{C}(\mathrm{MPa})$ & 900 \\
\hline$Y_{T}(\mathrm{MPa})$ & 27 \\
\hline$Y_{C}(\mathrm{MPa})$ & 200 \\
\hline$S_{x y}(\mathrm{MPa})$ & 80 \\
\hline$G_{I C}\left(\mathrm{KJ} / \mathrm{m}^{2}\right)$ & 220 \\
\hline
\end{tabular}
element model would be too cumbersome to use in progressive failure analyses. To maintain a reasonable number of elements and yet accurately account for failures in each of the plies, a two-level procedure is followed. In the first level, within each finite element, the material properties of the plies are homogenized. In the second level, within the progressive failure analysis, the stress and failure state of each ply is evaluated. The details of this procedure are described below. 
Elements of classical lamination theory (CLT) were used to construct and deconstruct the homogenized material properties and to evaluate ply-level values in a manner that is suitable for the PFA, but its use for this problem requires several assumptions:

1) Nominal percentages and uniform spatial distribution of $0^{\circ}, \pm 45^{\circ}$, and $90^{\circ} / 0^{\circ}$ plies at every quadrature point in each element of the model

2) No non-zero coupling (i.e., the 16,26 , and $B$-matrix) terms after ply failure

3) Bending deformations that are inherent in the CLT are not explicitly modeled. Rather, the deformations are modeled using solid elements

4) Independent material properties at each quadrature point in the element that can be degraded independently

5) Woven fabric can be treated as 2 plies of tape

Plies of each of the orientations are distributed nearly uniformly throughout the lug adding credibility to the assumption of a uniform spatial distribution of plies. Additionally, the large number of plies in the lug tends to reduce the effect of the coupling terms. The assumption of piecewise constant bending is reasonable given the number of integration points through the thickness of the lug and the relatively low bending gradient. The assumption of independence of properties at each quadrature point has been explored extensively for PFA analyses [Averill, 1992].

Prediction of failure within textile-based composite materials has been a topic of considerable attention for two decades [Poe and Harris, 1995; Glaessgen et al., 1996]. However, there is no accurate method for predicting the micromechanical details of damage progression in textile-based composites that has the computational efficiency needed to predict failure in structural models of the size used in this accident investigation. This deficiency in the state-of-the-art led to the approximation of the 8-harness satin weave material as plies of "equivalent" tape as shown in Figure 6. Hashin's failure criteria was used to predict failure of the equivalent tape.
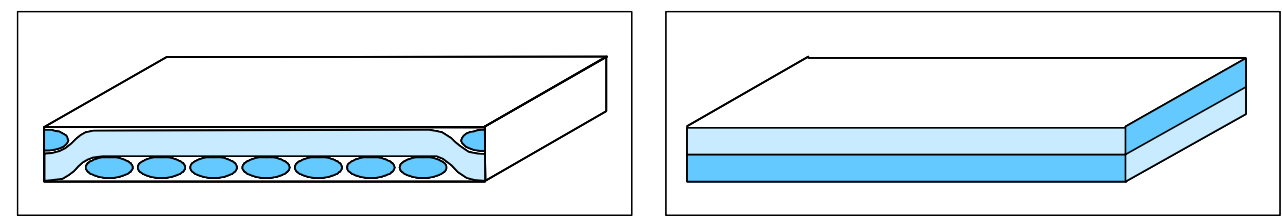

Figure 6. Eight-Harness Satin Weave and Tape Approximation.

\section{Contact}

Although most of the load transfer between the pin and lug is normal to the interface (initially, the global $x z$ plane), only friction prevents the pin from sliding (rigidly translating) in the global $y$-direction. Because of the proximity of the location of the material failures to the location of the pin-lug interface, considerable effort was taken to accurately model the details of the load transfer between the pin and lug.

Although the ABAQUS code correctly models the normal contact between the pin and lug, the modeling of friction along the pin-lug contact region was not straightforward. The lug analysis team did not have access to friction data about the lug, so the following approach was developed. A multi-point constraint (MPC) equation was generated to prevent sliding of the pin. In the MPC equation, the displacement of the pin in the global $y$-direction $\left(v_{P}\right)$ is set equal to the average of the global $y$-displacements of all of the nodes in the two rings on the lug hole $\left(v_{I}\right.$ and $v_{O}$ for average displacements of the inner and outer rings, respectively) shown in Figure 7. This MPC equation, referred to as Y-MPC \#1, was used for all analyses prior to the 2003-subcomponent test. Differences were found between the global-local moments computed by NASA using Y-MPC \#1 and the moments computed by Airbus using their global-local analysis process. The NASA lug team re-evaluated the MPC equation and concluded that it was not accurately simulating the global $y$-force

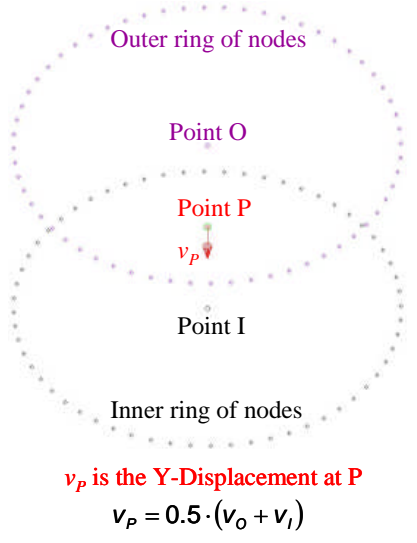

(a) Y-MPC \#1

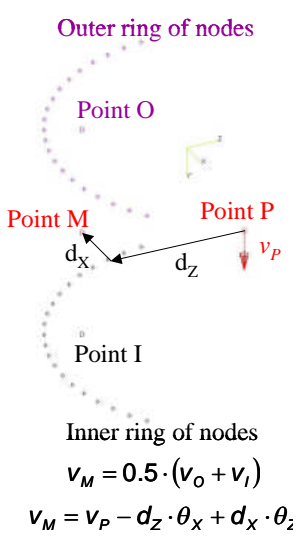

(b) Y-MPC \#2

\section{Figure 7. Multi-Point Constraint Formulation.}


reacted by the pin because the $y$-force can only react on the contact surface, and Y-MPC \#1 effectively treated the $y$ force as reacting around $360^{\circ}$ of the hole. In order to improve the simulation, another MPC equation, Y-MPC \#2, was developed.

For equation Y-MPC \#2, two $120^{\circ}$ arcs $\left( \pm 60^{\circ}\right.$ relative to the load vector) were used instead of the $360^{\circ}$ rings, as shown in Figure $7 \mathrm{~b}$. The average displacement of these two arcs is represented by the displacement $\left(v_{M}\right)$ at Point $\mathrm{M}$. The displacement at Point $\mathrm{M}$ is related to the pin displacement $\left(v_{P}\right)$ by an equation that includes the global $x$ - and $z$ rotations of the pin, as shown in Figure 7b. All lug results generated before the 2003-subcomponent test used YMPC \#1; all later analyses used Y-MPC \#2.

\section{A. Progressive Failure Analysis (PFA)}

\section{Background to Failure Theories}

Strength-based approaches for the prediction of initial and progressive failure in polymeric matrix composites are founded on a continuum representation of ply-level failure mechanisms. The comparative simplicity of applying strength-based criteria for the prediction of failure events within common analysis frameworks such as finite element procedures has led to this approach becoming increasingly accepted as a method for predicting the onset and development of material failure in composite structures.

Active research is directed towards representing micromechanical-level damage mechanisms in macroscopic, continuum-based failure criteria. These investigations have commonly elicited controversial discussions regarding the theoretical validity of developed failure criteria [Soden and Hinton, 1998a and b]. At issue is the difficulty of simulating the complexity of underlying failure mechanisms in terms of a discrete set of fixed strength parameters and the validity of using these parameters determined for individual lamina in the elastically constrained environment of an assembled laminate. The need to develop computationally efficient methodology to avoid detailed micromechanical analyses is aptly expressed by a passage by Hashin [Hashin, 1980]: "The microstructural aspects of failure are of such complexity that there is little hope of resolution of this problem on the basis of micromechanics methods. Such methods would require analytical detection of successive microfailures in terms of microstress analysis and microfailure criteria and prediction of the coalescence of some of them to form macrofailures which is an intractable task."

A large number of continuum-based criteria have been derived to relate internal stresses and experimental measures of material strength to the onset of failure [Rowlands, 1984; Nahas, 1986]. However, the use of any of these criteria for predicting failure beyond initiation may become theoretically invalid due to the underlying physics of interacting failure mechanisms that are implicitly neglected in the experimental determination of critical strength parameters.

2. Failure Theory Used in the PFA

In the analysis of the right rear composite lug, the Hashin criterion [Hashin, 1980] was chosen. Hashin's criterion assumes that the stress components associated with the plane of fracture control the failure. This consideration leads to the following equations expressing fiber and matrix failure written for general threedimensional states of stress.

Tensile fiber mode

$$
\left(\frac{\sigma_{11}}{X_{T}}\right)^{2}+\frac{1}{S_{x y}^{2}}\left(\sigma_{12}^{2}+\sigma_{13}^{2}\right)=1
$$

or

$$
\sigma_{11}=X_{T}
$$

Compressive fiber mode

$$
\left|\sigma_{11}\right|=X_{C}
$$

Tensile matrix mode $\left(\sigma_{22}+\sigma_{33}\right)>0$ 


$$
\frac{1}{Y_{T}^{2}}\left(\sigma_{22}+\sigma_{33}\right)^{2}+\frac{1}{\tau_{T}^{2}}\left(\sigma_{23}^{2}-\sigma_{22} \sigma_{33}\right)+\frac{1}{S_{x y}^{2}}\left(\sigma_{12}^{2}+\sigma_{13}^{2}\right) \geq 1
$$

Compressive matrix mode $\left(\sigma_{22}+\sigma_{33}\right)<0$

$$
\frac{1}{Y_{c}}\left[\left(\frac{Y_{c}}{2 \tau_{T}}\right)^{2}-1\right]\left(\sigma_{22}+\sigma_{33}\right)+\frac{1}{4 \tau_{T}^{2}}\left(\sigma_{22}+\sigma_{33}\right)^{2}+\frac{1}{\tau_{T}^{2}}\left(\sigma_{23}^{2}-\sigma_{22} \sigma_{33}\right)+\frac{1}{S_{x y}^{2}}\left(\sigma_{12}^{2}+\sigma_{13}^{2}\right) \geq 1
$$

In equations 1 to 5 , the strength values $\left(X_{T}, X_{C}, Y_{T}, Y_{C}\right.$, and $\left.S_{x y}\right)$ are defined in Table 1 . Note that both the normal stress in the fiber-direction, $\sigma_{11}$, and the shear stress components parallel to the fiber direction, $\sigma_{12}$ and $\sigma_{13}$, are considered in equation 1. In equations $1-5, \tau_{T}$ is the transverse shear strength corresponding to the $\sigma_{23}$ stress component, while $S_{x y}$ is the shear strength corresponding to the $\sigma_{13}$ and $\sigma_{12}$ components.

\section{Internal State Variable Approach}

Once failures are detected at a quadrature point, the material properties are degraded using an internal state variable approach. This approach degrades the properties from their original values to very small but non-zero values in a pre-determined sequence over several load steps. Material properties are degraded according to the particular active failure mode as determined by the Hashin criterion. For example, a compressive matrix mode failure requires that the matrix-dependent properties be degraded, but that the fiber-dependent properties, e.g. $\mathrm{E}_{11}$, remain unchanged. In these analyses, the strength values presented in Table 1 are used.

4. Progressive Failure Analysis Algorithm

Figure 8 shows the algorithm that is implemented as a user defined material (UMAT) subroutine within ABAQUS. Note that this algorithm consists of a preprocessing phase in which ply-level stresses are computed, an evaluation phase in which failures are determined, a material degradation phase in which ply level properties are degraded, and a post-processing phase in which updated laminate properties are computed. This algorithm is called for every quadrature point of every hexahedral element within the model, and updated material properties are evaluated at the quadrature points when the ply failure criteria are satisfied.

There are two adjustable parameters in this algorithm: the degradation schedule and the load (or displacement) increment. Studies undertaken by the authors have shown that a degradation factor of 0.7 (instead of 1.0 or $100 \%$ ) appears to be ideal for the stability of the algorithm. Rather than incrementing the loads, the current PFA increments

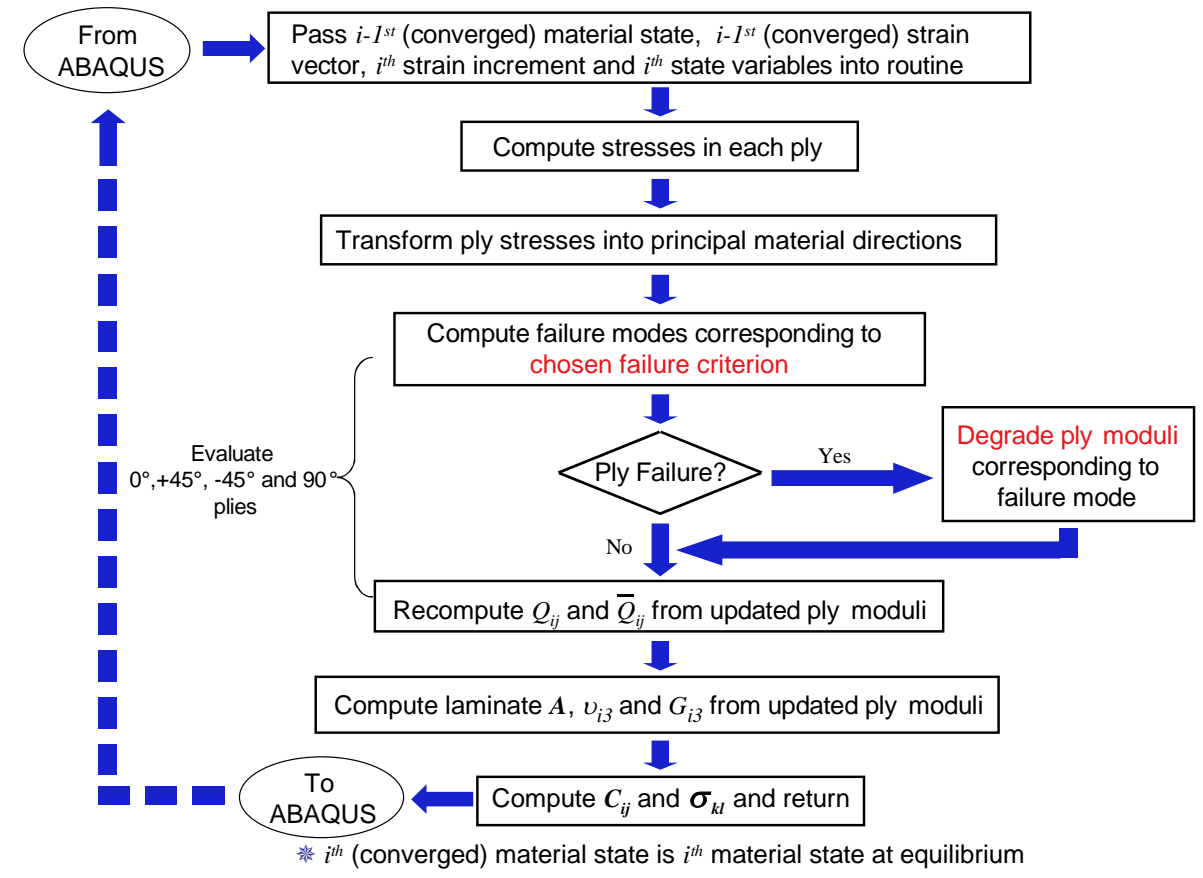

Figure 8. PFA Algorithm Used as a UMAT Subroutine in ABAQUS (Note: Stop is executed in ABAQUS and hence is not shown in this figure). 
the displacements and hence simulates displacement-controlled tests. This approach simplifies the process of simulating unloading past the peak load as discussed in the following section.

\section{5. "Load-Increment in the PFA"}

Displacement control is used in the current implementation of the progressive failure analysis to ensure that both the loading and unloading are traced by the algorithm. A load control procedure will encounter convergence difficulties after damage occurs because the monotonically increasing load applied to the damaged structure will cause abrupt failure. In contrast, a displacement-controlled procedure has fewer convergence difficulties after damage initiates because the load can decrease as damage forms, and the material becomes more compliant.

In cases where the maximum linear load, $P_{\max }$, carried by the specimen is known, the corresponding maximum linear displacement, $d_{\max }$, is calculated from a linear analysis. If $P_{\max }$ is unknown, a projected value is assumed and the corresponding maximum linear displacement, $d_{\max }$, is also calculated from a linear analysis. The displacements are incremented using the $d_{\max }$ as a guide and are termed here as load factor $\left(d / d_{\max }\right)$. A schematic of the load vs. load factor curve is shown in Figure 9. The solid line with symbols and dashed line represent a hypothetical PFA loaddisplacement curve and a linear load-displacement curve, respectively. Note that the load factor of unity will intersect the dashed line at $P_{\max }$, the maximum linear load, and corresponds to the maximum linear displacement, $d_{\max }$ (i.e. at load factor equal to unity). Once damage is determined and the corresponding material properties are degraded, the actual loaddisplacement curve will begin to deviate from the linear curve. The load continues to increase monotonically until a peak value, the failure load, $P_{\text {failure }}$, is reached. Then, $P$ decreases until a zero-value of load is reached or the analysis can no longer converge.

Note that in the PFA implementation, large displacement increments are chosen to start the algorithm, and shortly before damage initiates, the increment size is scaled down. As the damage accumulates, near the failure load, the increment size is scaled down further. The determination of the load factor increments is an art and requires the insight of an experienced analyst.

\section{Layered-Shell Model}

In addition to the coarse 3D element analyses of the AA 587 right rear lug described in the previous section, an analysis based on a layered-shell model was developed. The layered-shell analyses were developed as an alternate means of predicting the failure of the lug. The term layered-shell signifies that the thickness of the lug is modeled by several layers of shell elements rather than a number of layers of solid elements. The layered-shell analyses lend themselves to the evaluation of delamination initiation or propagation through the addition of decohesion elements between the shell layers. The analysis was developed in ABAQUS, and UEL and USFLD user-written subroutines were used for modeling the progressive delamination and intra-ply damage, respectively.

As with the solid-shell models, the layered-shell model was developed by modifying the original Airbus model of the right rear lug. The original Airbus model used 3D solid elements in the lug region and solid and shell elements in the remainder of the model. To develop the ABAQUS model, the faces of the solid elements in the $x z$ plane were converted into quadrilateral shell elements, and then the solid elements were converted into decohesion elements. The layered-shell model had 21000 nodes involving approximately 130000 equations.

\section{A. Material Modeling}

\section{Modeling Damage with Superimposed Shell Elements}

The layered-shell models use a novel and computationally efficient element superposition technique that separates the failure modes for each ply orientation and does not rely on the computation of the $([A],[B]$, and $[D])$ matrices [Dávila et al., 2000]. The modeling is performed such that the elements in the region around the bolt hole, 
where a potential for damage growth is anticipated, are constructed of four superposed layers of shell elements that share the same nodes. No centroidal offset is applied to any of the elements. Each layer of elements represents one ply orientation ( 0 or 45 or -45 or 90

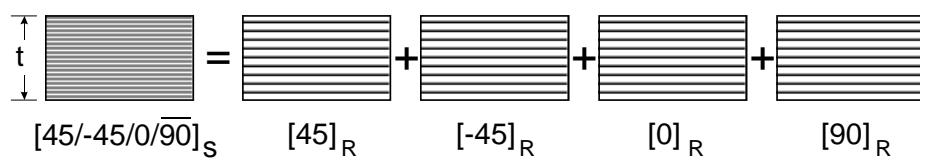
degrees), and each element spans the entire thickness of the laminate as shown in Figure 10. It is implied that the plies for each orientation are uniformly distributed and can be smeared over the thickness of the laminate. The elements used in the analyses consist of the ABAQUS four-node reduced-integration shear deformable S4R element [ABAQUS, 2000].

To model the appropriate stiffnesses corresponding to a given damage state, reduced engineering properties are applied to each layer. A reduced material property for a given orientation is simply the product of the engineering property and the sum of the thicknesses of all the plies in that orientation divided by the total laminate thickness. Reduced material properties are denoted by the notation []$_{R}$, as illustrated in Figure 10 . Bending effects are taken into account by the use of five integration points through-the-thickness of the laminate.

\section{B. Progressive Failure Analysis for the Layered-Shell Model}

A progressive damage model for notched laminates under tension was first proposed by Chang et al. [Chang and Chang., 1987] and accounts for all of the possible failure modes in each ply except delamination. Chang and Lessard [Chang and Lessard, 1991] later investigated the damage tolerance of composite materials subjected to compressive loads. The present analysis, which also deals with compression loads, is largely based on the work by Chang and Lessard. However, the present analysis extends Chang's method from two-dimensional membrane effects to a shell-based analysis that includes bending.

The failure criteria applied in the present analysis are those for unidirectional fiber composites as proposed by Hashin [Hashin and Rotem, 1973], with the elastic stiffness degradation models developed for compression by Chang and Lessard [Chang and Lessard, 1991]. Unidirectional failure criteria are used, and the stresses are computed in the principal directions for each ply orientation. The failure criteria included in the present analysis are summarized below. In each, failure occurs when the failure index exceeds unity.

- Matrix failure in tension and compression occurs due to a combination of transverse stress $\sigma_{22}$ and shear stress $\sigma_{12}$. The failure index $e_{m}$ can be defined in terms of these stresses and the strength parameters $Y_{T / C}$ and the shear allowable $S_{x y}$. The matrix allowable $Y_{T / C}$ takes the values of $Y_{T}$ in tension and $Y_{C}$ in compression. Failure occurs when the index exceeds unity. Assuming linear elastic response, the failure index has the form:

$$
e_{m}=\sqrt{\left(\frac{\sigma_{22}}{Y_{T / C}}\right)^{2}+\left(\frac{\sigma_{12}}{S_{x y}}\right)^{2}}
$$

- Fiber buckling/tension failure occurs when the maximum compressive stress in the fiber direction exceeds the fiber tension or buckling strength $X_{T / C}$, independently of the other stress components. The failure index for this mechanism has the form:

$$
e_{b}=\frac{\left|\sigma_{11}\right|}{X_{T / C}}
$$

- Fiber-matrix shearing failure occurs due to a combination of fiber compression and matrix shearing. The failure index has the form:

$$
e_{f}=\sqrt{\left(\frac{\sigma_{11}}{X_{T / C}}\right)^{2}+\left(\frac{\sigma_{12}}{S_{x y}}\right)^{2}}
$$

The finite element implementation of this failure analysis was developed in ABAQUS using the USFLD userwritten subroutine. The program calls this routine at all material points of elements that have material properties 
defined in terms of the field variables. The routine provides access points to a number of variables such as stresses, strains, material orientation, current load step, and material name, all of which can be used to compute the field variables. Stresses and strains are calculated at each incremental load step and evaluated by the failure criteria to determine the occurrence of failure and the mode of failure.

\section{Global-Local Analysis}

\section{A. Global-Local Connection Procedure}

The aerodynamic loads on the vertical tail at failure (during the accident) were computed by Airbus and provided to NASA. This load case, referred to as W375, was directly applied only to the global model. The local region of the global NASTRAN (MSC/NASTRAN, 1997) model is shown in Figure 11a. Because the global model is a MSC/NASTRAN model and the local lug model (the coarse 3D model) is an ABAQUS model, it was not possible to embed the local model in the global model. Conversion of the NASTRAN model to ABAQUS was not feasible due to time constraints. Additionally, the version of NASTRAN used for the global model was not capable of modeling contact. The details of the global model and global analysis are discussed by Young et al. [Young et al., 2005].

Along the interfaces between the global and local models, the continuity of the displacements and the reciprocity of tractions need to be satisfied. An iterative process was developed to ensure satisfaction of these requirements. This process is illustrated in Figure 12 and is implemented as follows:

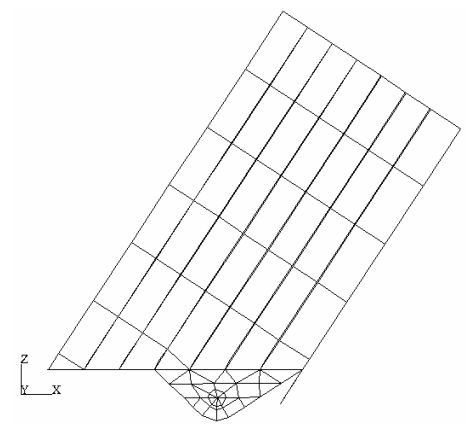

(a) Local Region in Global Model

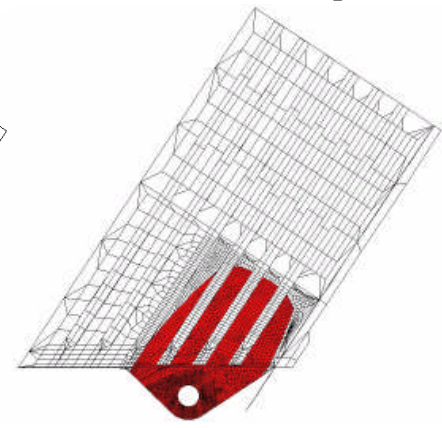

(b) Local Model with Transition Mesh

Figure 11. Models of Region Near Right Rear Lug.

1) Perform the global analysis using the global model and evaluate the displacements at all the nodes in the global model. Let $\left\{u_{G}\right\}$ represent the displacements of the global nodes along the global-local boundary and $\left\{u_{L}\right\}$ represent the displacements of the local nodes along the global-local boundary. Evaluate the tractions at the global nodes, $\left\{F_{G}\right\}$, from the elements that are entirely in the global region. That is, evaluate the tractions that do not include the elements that occupy the local region of the global model.

2) Establish a transformation matrix, $[T]$, between $\left\{u_{G}\right\}$ and $\left\{u_{L}\right\}$, and use this matrix to compute $\left\{u_{L}\right\}$ using

$$
\left\{u_{L}\right\}=[T]\left\{u_{G}\right\}
$$

3) Solve the local model with $\left\{u_{L}\right\}$ as prescribed displacements.

4) Because of the prescribed displacements, reactions at the interface nodes in the local model $\left\{R_{L}\right\}$ are produced.

5) Local reactions are mapped back to the global nodes using

$$
\left\{R_{G}\right\}=[T]^{T}\left\{R_{L}\right\}
$$

Equation 10 is obtained by requiring that the work done on the global-local boundaries in the local model $(1 / 2) \cdot\left(\left\{u_{L}\right\}^{\mathrm{T}} \cdot\left\{R_{L}\right\}\right)$ and the global model $(1 / 2) \cdot\left(\left\{u_{G}\right\}^{\mathrm{T}} \cdot\left\{R_{G}\right\}\right)$ are identical. The $\left\{R_{G}\right\}$ reactions represent the stiffness of the local model in the global model.

6) The global tractions $\left\{F_{G}\right\}$, in general, will not be identical to the reactions mapped from the local model, $\left\{R_{G}\right\}$, as the reciprocity of tractions is not imposed. Thus, a residual, $\{\mathrm{r}\}$, is left on the global-local boundary:

$$
\{r\}=\left\{F_{G}\right\}-\left\{R_{G}\right\}
$$

7) Evaluate a norm $\|r\|$ for the residual $\{r\}$ using 


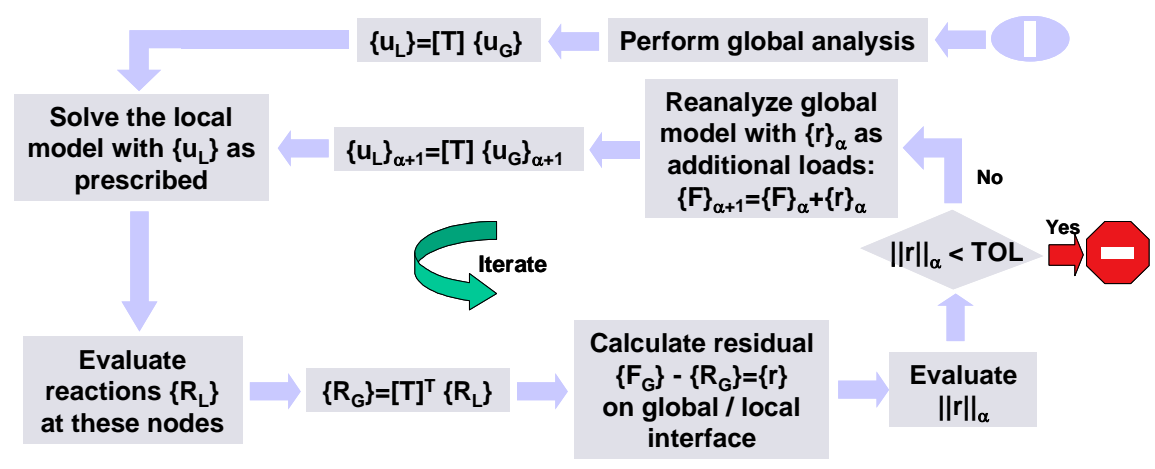

Figure 12. Global-Local Iterative Analysis Process.

$$
\|r\|_{\alpha}=\frac{\sum_{\text {Boundary }}\left|\left(F_{G_{i}}\right)_{\alpha}\left[\left(u_{G_{i}}\right)_{\alpha}-\left(u_{G_{i}}\right)_{\alpha-1}\right]\right|}{\sum_{\text {Boundary }}\left|\left(F_{G_{i}}\right)_{\text {Initial }}\left(u_{G_{i}}\right)_{\text {Initial }}\right|}
$$

where $\alpha$ is the current iteration number in the convergence process and the $\Sigma$ implies accumulation at all nodes on the global-local boundary.

8) If the normalized residual is less than a prescribed tolerance, then this procedure is stopped, and the system has converged. If the normalized residual is higher than the prescribed tolerance, then the residual vector is added as an initial load set in the global model and the global analysis is executed again (i.e. return to step 1).

The interfaces between the global and local model are defined on 9 edges (shown as red and blue lines in Figure 13) and at 17 discrete locations (shown as red dots in Figure 13). The coarse 3D model was modified so that the local edge nodes matched the global edge nodes exactly as shown in Figure 11b. Therefore, the mapping from the global and local models can be accomplished with a unit $[T]$ matrix.

To maintain symmetry of the global model, the stiffness of both the right rear and left rear lugs was updated by the global-local process. Thus, during the global-local process, two local analyses were performed during each iteration. Instead of creating another FEM, one local FEM was used for both the right rear and left rear lug. For the left rear lug, the loads and boundary conditions were mirrored about the global $x z$-plane (i.e. the sign of $F_{Y}, M_{X}, M_{Z}$, $v, \theta_{X}$, and $\theta_{Z}$ are reversed).

\section{B. Global-Local Analysis and PFA}

The global-local process described in the previous section assumes that the stiffness of the local model does not change in the iterative procedure. Similarly, the PFA assumes that the boundary conditions on the local model do not change as the PFA continues. The most rigorous analysis of the VTP requires that damage determined in the local model be returned to the global model. That is, at step 2 of the global-local process, the PFA needs to be performed to determine the current damage state of the lug. After convergence is obtained (and equilibrium is established), the global-local process is continued with step 3.

Such a rigorous procedure involving both the global model and the local model and with the current large degree of freedom model is impractical. Therefore, the global-local procedure is performed first to determine the boundary conditions on the global-local interfaces and the loads at the pin. With these boundary conditions and loading, the PFA is performed on the local model. The verification of this decoupling assumption is provided in the Results section.

Convergence of the forces and moments in the right rear lug for the W375 load case are plotted in Figures 14a and $14 \mathrm{~b}$, respectively. In these figures, the reactions are normalized by the average of the global and local results at the end of the sixth iteration. At the sixth iteration, the difference between the global and local forces is less than 1 $\mathrm{kN}$, and the difference between the corresponding moments is approximately $0.03 \mathrm{kN}-\mathrm{m}$. 


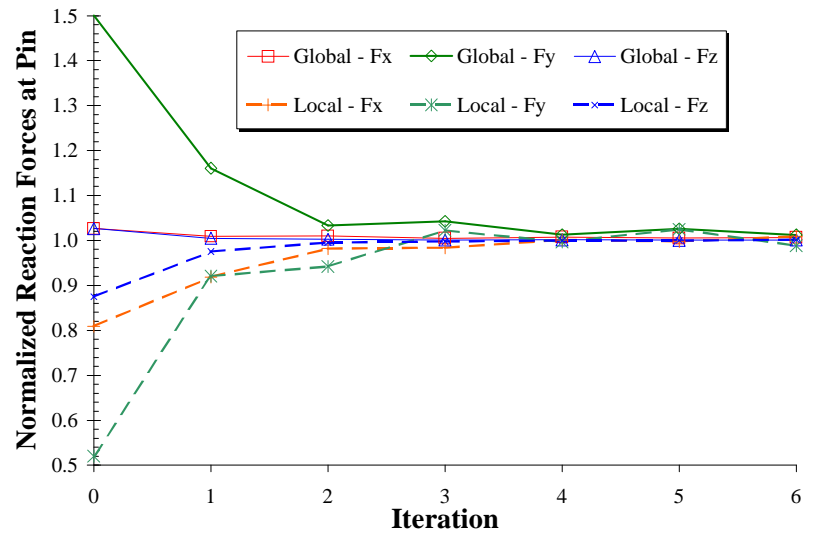

(a) Convergence of Pin Forces

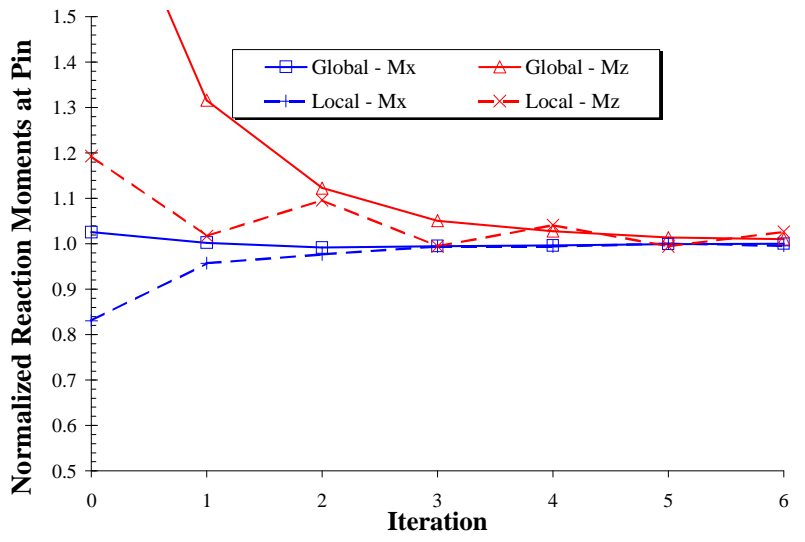

(b) Convergence of Pin Moments

Figure 14. Convergence in Global-Local Analysis (Load Case W375).

\section{Results}

The PFA results are compared with available experimental results for the 1985-certification test (X2/1 and X2/2 specimens) and the 2003-subcomponent (SC) test. In addition, the load case corresponding to W375 is analyzed using the coarse 3D model. Table 2 presents various load cases analyzed and the corresponding models used in the analysis. Note that all of the PFA analyses shown in Table 2 were performed considering both geometric nonlinearity and pin-lug contact.

Table 2. Various Load Cases Analyzed and Finite Element Models Used.

\begin{tabular}{|c|c|c|c|c|c|c|}
\hline \multirow{2}{*}{\multicolumn{2}{|c|}{ Finite Element Models }} & \multicolumn{5}{|c|}{ Load Cases Analyzed } \\
\hline & & $\mathbf{X} 2 / 1$ & $\mathrm{X} 2 / 2$ & PFA Studies & SC Test & W375 \\
\hline \multirow{3}{*}{ Solid-Shell Model } & Coarse 3D Model & & & $\mathbf{X}$ & & $\mathbf{X}$ \\
\hline & 1985 Test Model & & $\mathbf{X}$ & & & \\
\hline & SC Test Model & & & & $\mathbf{X}$ & \\
\hline Layered-Shell Model & & $\mathbf{X}$ & $\mathbf{X}$ & $\mathbf{X}$ & & \\
\hline
\end{tabular}

\section{A. 1985-Certification Test (X2/2 Specimen)}

\section{Configuration}

As part of the certification process for the composite lugs on the A300-600R aircraft, Airbus developed the certification test configuration shown in Figure 15. In this configuration, a hydraulic piston and lever were used to apply an in-plane load to the lug as shown in Figure 15a. The test specimen was fixed around the perimeter of the skin as shown in Figure 15b, and the constraint due to rib 1 was simulated using the transverse girder shown in Figure $15 \mathrm{c}$. Because all of the loading was in the plane of the specimen, the $M_{X}$ at the lug in this test was entirely due to the combination of $F_{X}, F_{Z}$, and the eccentricity. A boundary condition of $\theta_{X}=0$ at the pin is hypothesized and is used in the analysis.

The instrumentation on the X2/2 test specimen consisted of 16 strain gauges as shown in Figure 16. There are two sets of back-to-back rosettes on the tapered portion of the lug immediately above rib

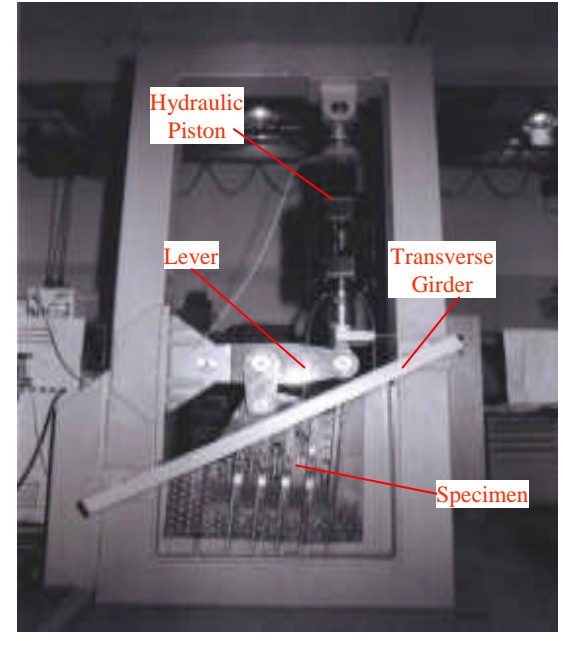

(a) Test Apparatus

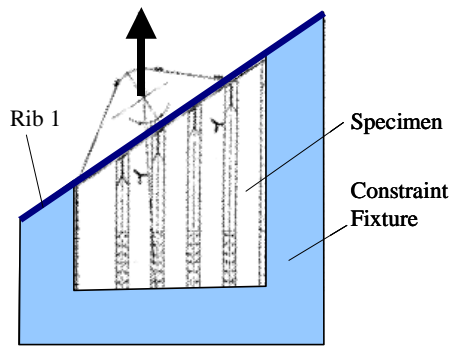

(b) Test Specimen

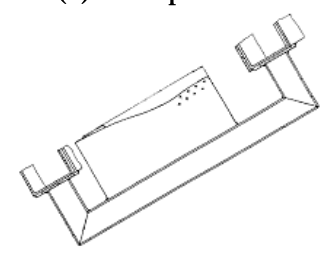

(c) Transverse Girder

Figure 15. 1985-Certification Test Configuration.

13

American Institute of Aeronautics and Astronautics 
1 (gauges 1-12) and four uniaxial gauges along the profile of the lug (gauges 13-16). During the test, all 16 gauges were monitored. The load vs. strain data from all these 16 gauges was available and was used in the PFA validation.

\section{Results}

Figure 17 shows the strain gauge results obtained from Airbus as open red circle symbols and NASA's finite element predictions made using the solid-shell model as solid blue lines. Applied load is shown in $\mathrm{kN}$ on the ordinate, and measured or predicted strain is shown (in thousands of microstrain) on the abscissa.

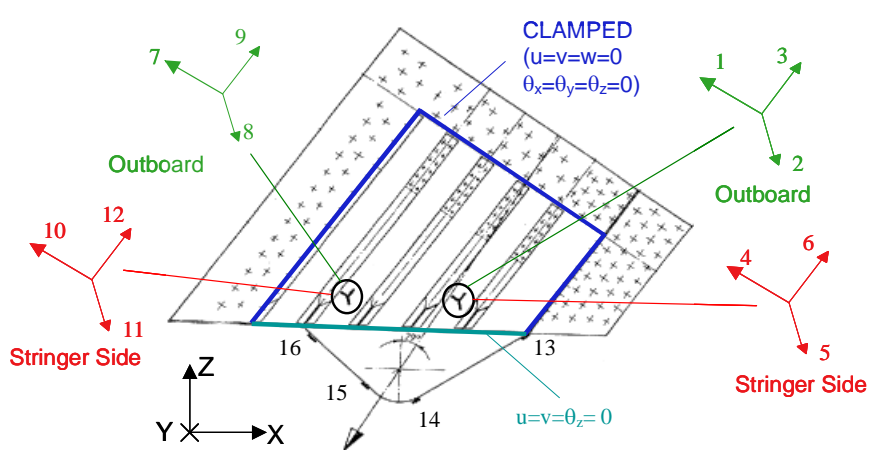

Figure 16. Strain Gauges on X2/2 Test Specimen.

Because gauges 13 and 16 are located near large changes in stiffness, they are not shown in Figure 17. In general, the predicted values agree very well with the strain gauge results. However, the predicted values do not agree well with strains from gauges 3 and 10. The reason for these two deviations is unknown. Also, because the location of gauges 14 and 15 through-thethickness was not known, finite element predictions of strain on the outboard side and stringer side of the lug are shown. These predictions bound the strain gauge results. From this figure, it was concluded that the present PFA represents accurately the behavior of the lug over the complete loading range.

The computed values of $F_{\text {Res }}$ (resultant of $F_{X}, F_{Y}$, and $F_{Z}$ force components) and $M_{X}$ vs. load factor are shown in Figure 18. In Figure 18 , the load factor is a nondimensional scaling factor that is applied to the displacements during the PFA analysis. A load factor of 1.0 corresponds to the displacements produced from a linear analysis. The curve for resultant force $\left(F_{\text {Res }}\right)$ vs. load factor is shown as a solid blue line with open circle symbols and the curve of $M_{X}$ vs. load factor is shown as a solid red line with open square symbols. The linearly projected values of $M_{X}$ and $F_{R e s}$ are shown as closed diamonds. The failure load from the $\mathrm{X} 2 / 2$ test specimen is shown as a thick horizontal red line. Peak values of $M_{X}$ and $F_{\text {Res }}$ are shown on the graph and in the tabular insert as points A and B, respectively. The load factor for the linear case and points A and B are shown with vertical dashed lines. The $F_{\text {Res }}$ at the maximum moment (Point A) agrees extremely well
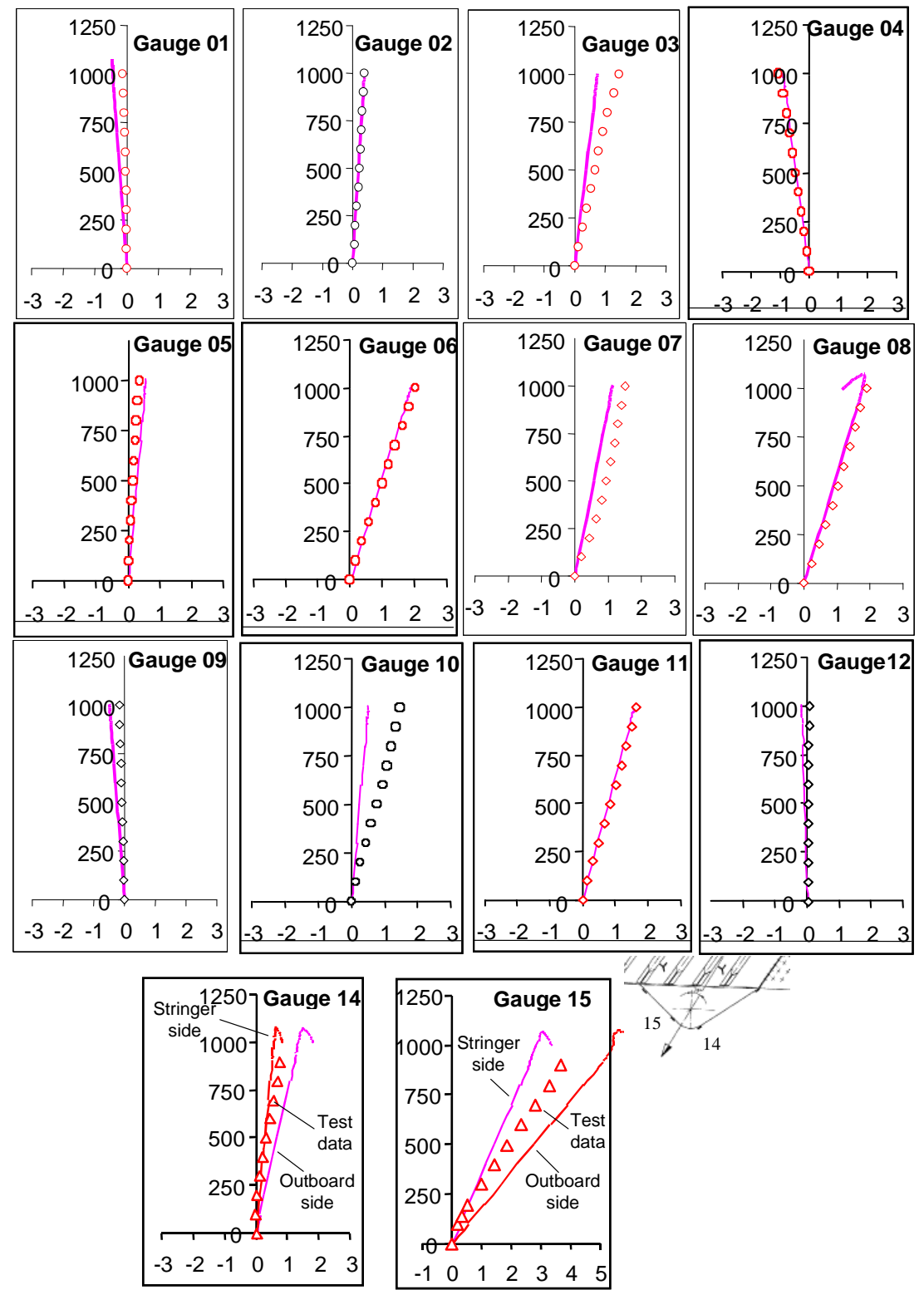

Figure 17. Strain Gauge and Finite Element Results.

14

American Institute of Aeronautics and Astronautics 


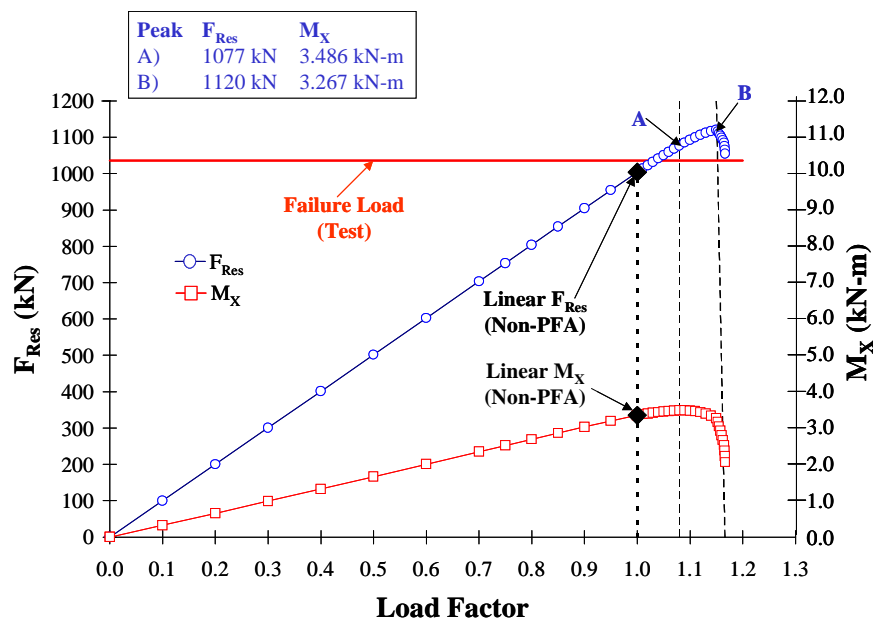

Figure 18. Load and Moment vs. Load Factor for 1985Certification Test.

with the experimentally determined value for this configuration. The extent of the damage predicted by the PFA in Figure 19 agrees well with that observed during the 1985-certification test shown in Figure 20. Note that Figure 19 is based on superposition of all active failure modes within all ply types at each Gauss point in the model.

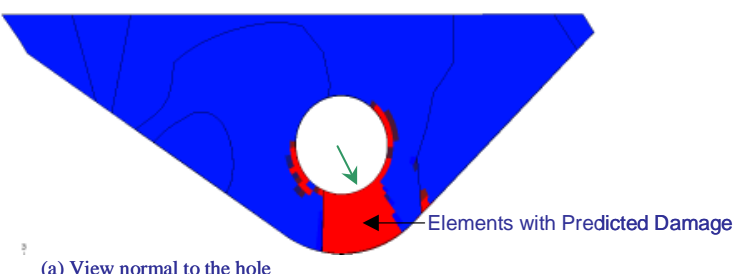

(a) View normal to the hole

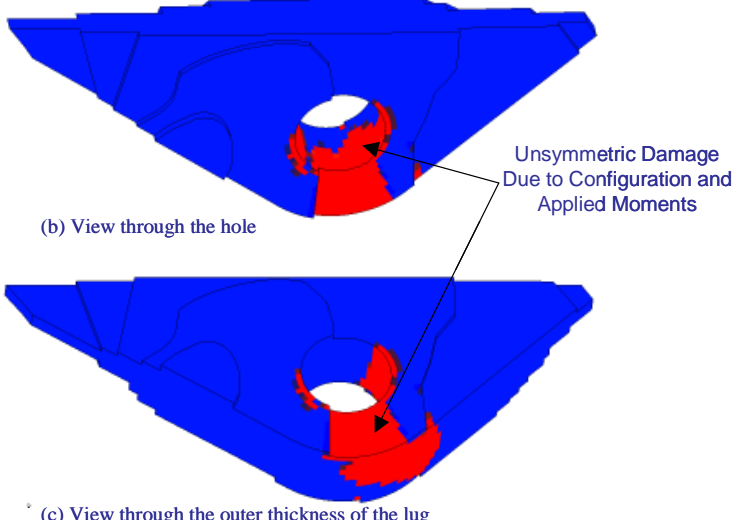

Figure 19. Damage Prediction from PFA for 1985-Certification Test.
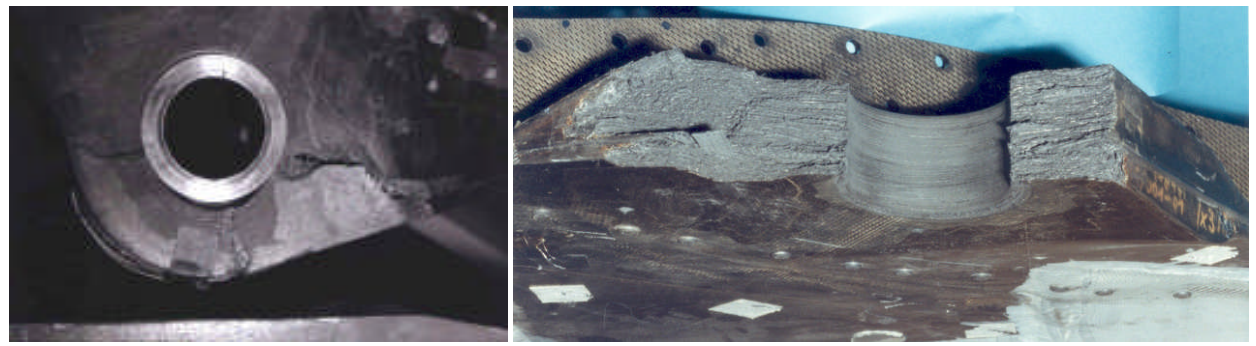

Figure 20. X2/2 Test Specimen - Observed Failure.

\section{B. 2003-Subcomponent Test}

As part of the AA 587 accident investigation, Airbus developed a new certification test configuration to more accurately simulate the load introduction and boundary conditions near the lug. The 2003-subcomponent (SC) test model and the PFA algorithm shown in Figure 8 were used to predict the response of the 2003-subcomponent test specimen with boundary conditions shown in Figure $5 \mathrm{~b}$. Because the exact value of the $M_{X}$ to be applied was unknown prior to the test, several values were considered as shown in Table 3. Note that in Table 3, because the SC test model is a left rear lug, the loads and moments are mirrored from their corresponding right rear lug load cases (i.e. the sign of $F_{Y}, M_{X}, M_{Z}, v, \theta_{X}$, and $\theta_{Z}$ are reversed). The pin forces in all cases in Table 3 correspond to the global-local analysis with Y-MPC \#1 (with $F_{Y}$ reversed). Case (C) was analyzed before the 2003 -subcomponent test and corresponds to an $M_{X}$ value of $6.537 \mathrm{kN}-\mathrm{m}$. Cases (D) and (E) were analyzed after the subcomponent test. Cases (D) and (E) correspond to the actual $\theta_{X}$ value of $0.51^{\circ}$ applied in the test with $360^{\circ}$ friction contact (Y-MPC $\# 1$ ) and $120^{\circ}$ friction contact (Y-MPC \#2), respectively. Post-test linear analyses gave the $M_{X}$ values of 6.67 and $6.27 \mathrm{kN}-\mathrm{m}$ for cases (D) and (E), respectively.

Because the PFA is implemented as a displacement- (translation and rotation) controlled process, a linearly projected target value of $M_{X}$ based on an assumed linear relationship between applied rotation and the resulting

Table 3. Pin Moments and Rotations for Subcomponent Test Model (left rear lug).

\begin{tabular}{|c|c|c|c|c|c|}
\hline Loading Case & $\boldsymbol{M}_{\boldsymbol{X}}$ & $\boldsymbol{M}_{\boldsymbol{Z}}$ & $\theta_{\boldsymbol{X}}$ & $\theta_{\boldsymbol{Y}}$ & $\theta_{\boldsymbol{Z}}$ \\
\hline SC Test W375 (C) & +6.537 & -1.000 & 0.487 & 0.000 & -0.065 \\
\hline SC Test W375 (D) & +6.670 & -0.379 & 0.510 & 0.000 & 0.000 \\
\hline SC Test W375 (E) & +6.270 & -0.508 & 0.510 & 0.000 & 0.000 \\
\hline
\end{tabular}

15

American Institute of Aeronautics and Astronautics 
moment was used. Note that as damage develops, the specimen loses its stiffness and hence will not carry the moment that is predicted by the linear relationship.

The computed values of $F_{R e s}$ and $M_{X}$ vs. load factor are shown for load cases SC (C), SC (D), and $\mathrm{SC}(\mathrm{E})$ in Figures 21a to 21c, respectively, for applied rotations resulting from linearly projected load and moment values as given in Table 3 . The curves for resultant force $\left(F_{\text {Res }}\right)$ vs. load factor are shown as solid lines with open circles, and the curves of $M_{X}$ vs. load factor are shown as solid lines with open square symbols. The linearly projected values of $M_{X}$ and $F_{R e s}$ are shown as closed diamonds. The failure load observed during the test is shown as a thick horizontal red line in Figures 21a to 21c. Peak values of $M_{X}$ and $F_{\text {Res }}$ are shown on the graph and in the tabular insert as points $\mathrm{A}$ and $\mathrm{B}$, respectively. The load factor for the linear case and points $\mathrm{A}$ and $\mathrm{B}$ are shown with vertical dashed lines.

Two entirely different loading sequences are represented by the sets SC (C) (Figure 21a) and SC (D) and (E) (Figures 21b and 21c). In load case SC (C), the translations and rotations were applied simultaneously and proportionally starting from zero values to develop the $F_{\text {Res }}$ and $M_{X}$ shown in the figures. For load cases SC (D) and (E), $\theta_{X}$ was applied initially until the desired initial rotation $\left(\theta_{X}\right)$ was reached, and then the translations and rotations were increased proportionally. These later cases (D and E) represent more accurately the loading sequence during the 2003-subcomponent test.

While the curves in Figures 21a to 21c show the same general trends, increased values of $M_{X}$ result in lower values of $F_{\text {Res }}$ at failure. Also, larger values of $M_{X}$ decrease the difference between $F_{\text {Res }}$ at peak moment (point A) and maximum $F_{\text {Res }}$ (point B). The difference between the values of points $A$ and $B$ is largest for load case SC (E) in which an initial value of $\theta_{X}$ is applied, and then is held constant. The constant rotation contributes to an artificial stiffening of the lug in load case SC (E) and results in higher peak $F_{\text {Res }}$ than for load case SC (C).

The damage predictions for the lug under load case SC (C) at peak moment and peak force are shown in Figures $22 \mathrm{a}$ and $22 \mathrm{~b}$, respectively. The mode of damage (cleavage type failure) is the same as seen previously in the 1985 -certification test. The extent of the damage predicted by the PFA (Figures $22 \mathrm{a}$ and 22b) also agrees well with that observed during the SC test shown in Figure 23. These damage surfaces are consistent with the damage surfaces seen in the other cases.

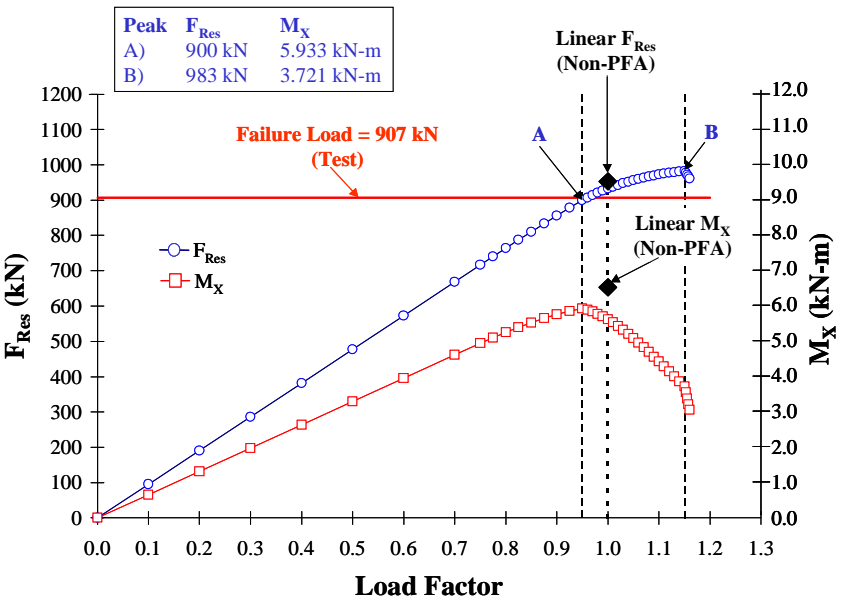

(a) SC (C) Load Case

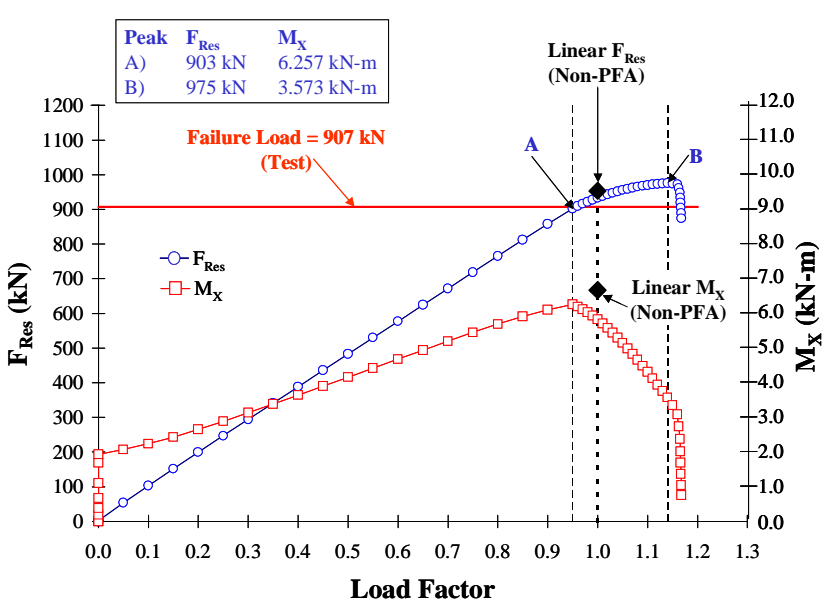

(b) SC (D) Load Case

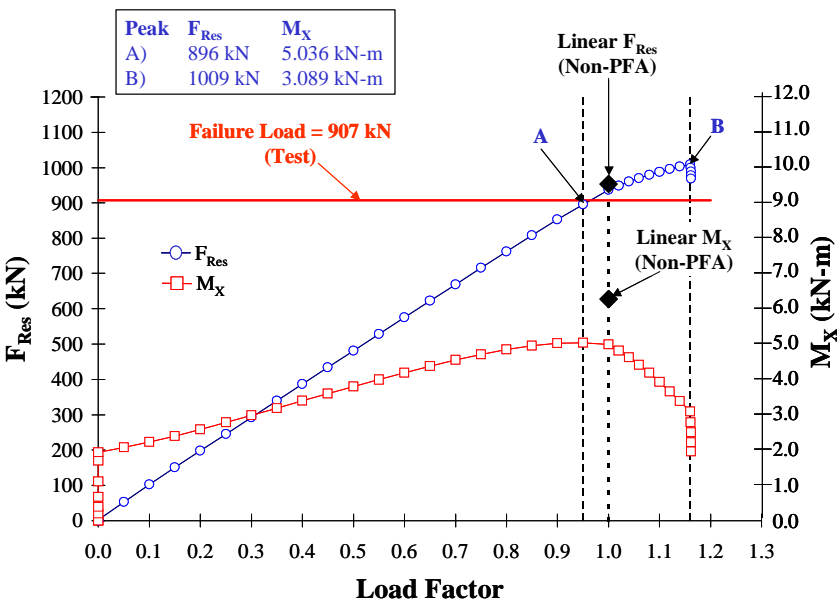

(c) SC (E) Load Case

Figure 21. Load and Moment vs. Load Factor. 


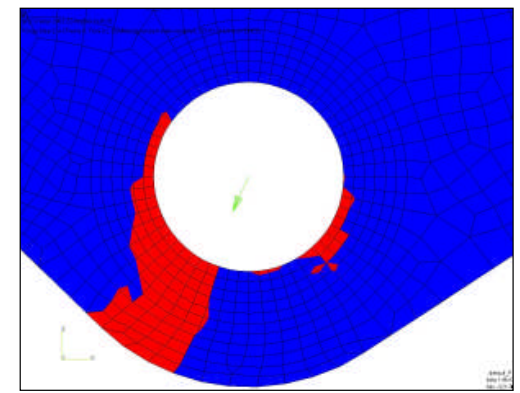

(a) Damage Region at Peak Moment

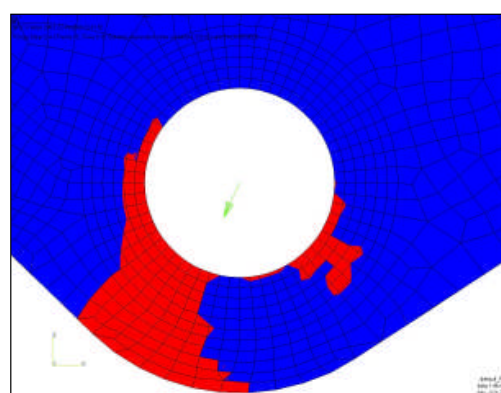

(b) Damage Region at Peak Force

Figure 22. Damage Regions for SC (C) Load Case.

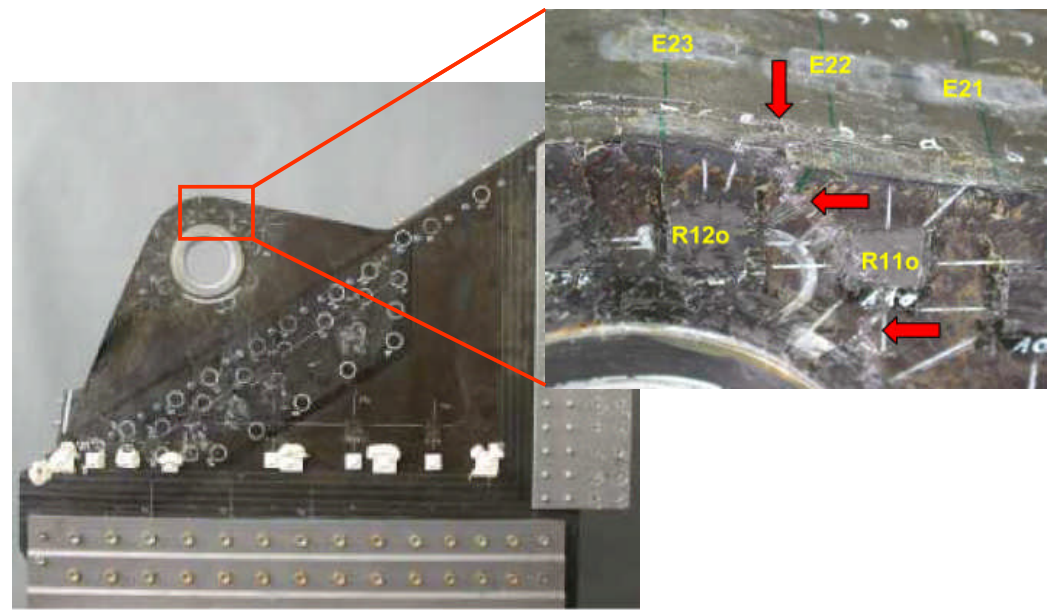

Figure 23. 2003-Subcomponent Test - Observed Failure (Red arrows point to the primary fracture path).

\section{W375 Accident Case PFA Analysis}

The forces and moments at the pin and the boundary conditions on Table 4. Pin Rotations for Load Case the global-local interfaces for W375 accident case were obtained from the global-local analysis. The corresponding pin rotations predicted from global-local analysis are given in Table 4 and are $48 \%$ higher than those used in the Airbus 2003-subcomponent test because they represent

\section{W375 in Accident Model (RHS).}

\begin{tabular}{|c|c|c|}
\hline CASE & $\theta_{X}$ & $\theta_{Z}$ \\
\hline Accident W375 & 0.756 & 0.286 \\
\hline
\end{tabular}
global rotations and include the effect of the rotation of the fuselage; the boundary conditions during the test did not consider the deformation of the fuselage and corresponded to a fixed condition at the base of the VTP.

The computed values of $F_{R e s}$ and $M_{X}$ vs. load factor are shown for the W375 accident case in Figure 24, using

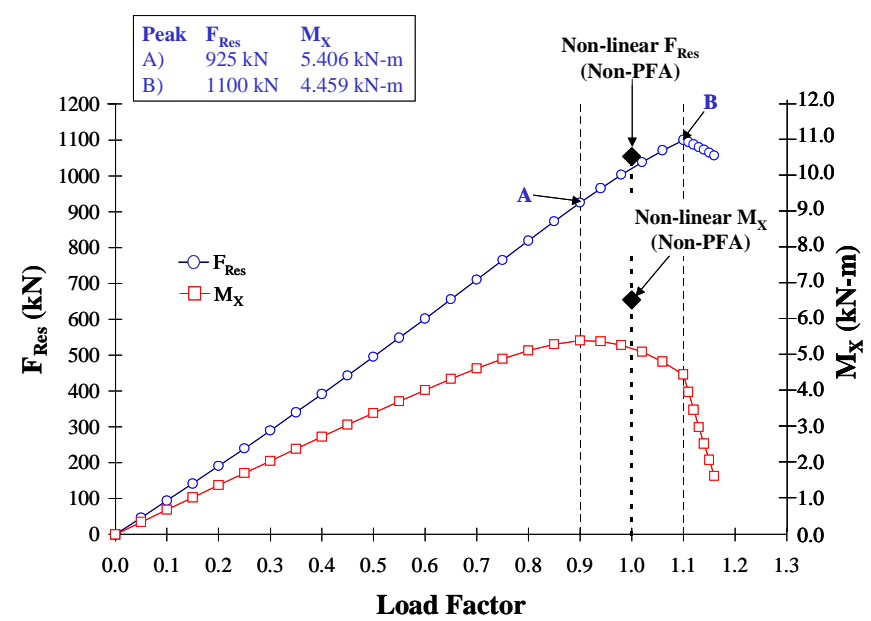

Figure 24. Load and Moment vs. Load Factor for W375 Load Case. applied translations and rotations resulting from linearly projected load and moment values. The curve for resultant force $\left(F_{\text {Res }}\right)$ vs. load factor is shown as a solid blue line with open circle symbols, and the curve of $M_{X}$ vs. load factor is shown as a solid red line with open square symbols. The linearly projected values of $M_{X}$ and $F_{R e s}$ are shown as closed diamonds. Peak values of $M_{X}$ and $F_{R e s}$ are shown on the graph and in the tabular insert as points $\mathrm{A}$ and $\mathrm{B}$, respectively. Further, the extent of the damage predicted by the PFA for the W375 accident case (Figure 25), again a cleavage type failure, generally agrees with the damage seen in a photograph of the failed AA 587 right rear lug in Figure 26. These damage predictions are similar to those obtained for the 1985-certification test and the 2003-subcomponent test. 

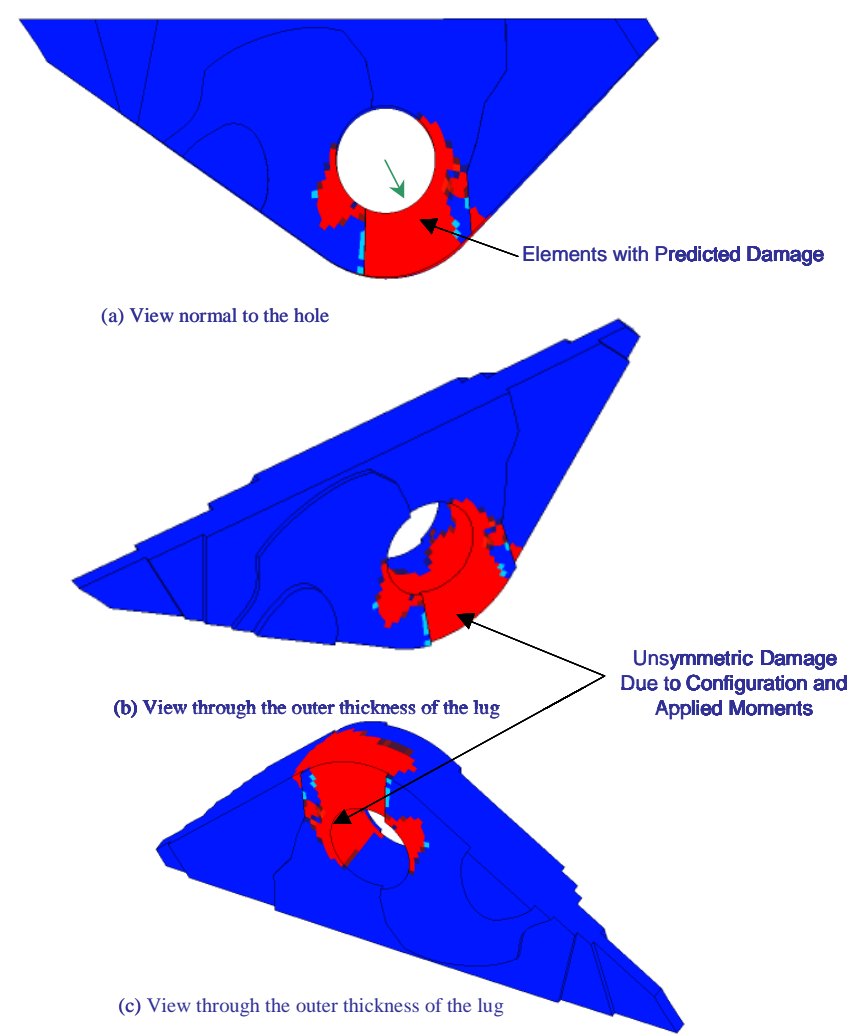

Figure 25. Damage Prediction for W375 Accident case from PFA.

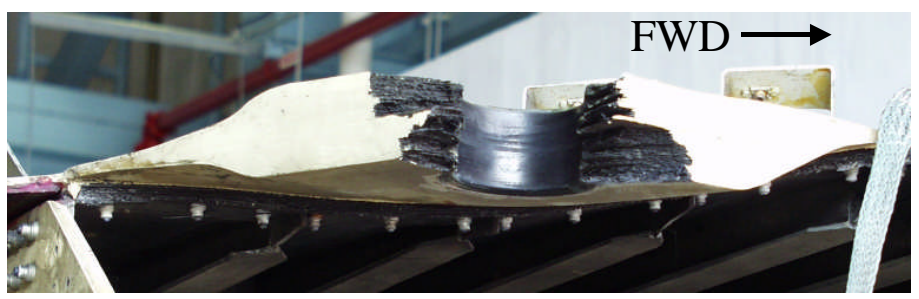

Figure 26. AA 587 Right Rear Lug - Observed Failure.

\section{Discussion}

This section discusses the results and lessons learned during the course of the analysis of the failure of the AA 587 right rear lug.

\section{A. Effect of $M_{X}$ and $M_{Z}$ on Experimentally Determined Failure Load}

As discussed in the Results section, the moment $M_{X}$ has a significant effect on the failure loads during the tests. Larger absolute values of $M_{X}$ result in lower failure loads for the lugs. For example, an observed increase in $M_{X}$ of 45 percent from the 1985-certification test (Figure 18) to the 2003-subcomponent test (Figure 21c) caused a 17 percent decrease in the failure load. In contrast, the moment $M_{Z}$ was determined to have a marginal effect on the failure load.

\section{B. Failure Modes}

The classical failure modes of a bolted joint are bearing failure, net tension failure, and shear-out failure. In addition to these three classical modes of failure, a failure identified as cleavage failure is also common [Camanho and Matthews, 1999]. The progressive failure analysis showed that the right rear lug failures are very similar to the cleavage type, but do not show separation of the failed piece from the remainder of the lug. Ideally, the progressive failure analysis of a lug should reproduce the entire sequence of failure events and should end with an analysis result exhibiting the same fracture surfaces as those on the failed part. However, several issues in the analysis make the determination of the fracture sequence difficult. The first issue pertains to the convergence of the numerical solution. Once the ultimate strength of the lug is exceeded, the lug is no longer in equilibrium and the numerical procedure fails to yield a converged solution. Secondly, models assume that all the applied loads and boundary displacements are incremented proportionally to each other during the analysis. The proportionality is a reasonable assumption until the ultimate strength is exceeded. After the peak force, the stiffness of the lug changes dramatically, and the assumption of load proportionality is no longer valid. Finally, damage is modeled as a softening of the material continuum rather than as a stress free surface or crack. Consequently, fracture surfaces that are plainly observable in the failed part are not as clearly represented in the model.

\section{Test and Accident Case Comparisons}

Figures 27 and 28 compare the failure loads and $M_{X}$ variation predicting with the solid-shell model and PFA for the three cases: the 1985-certification test, 2003-subcomponent (SC) test, and the W375 accident condition. The stiffnesses of the lug (represented by the slope of the $F_{\text {Res }}$ vs. Load Factor curve in Figure 27) for the three cases and the maximum moment $M_{X}$ (Figure 28) for the SC test and the W375 accident case agree very well.

The failure loads (Figure 29) and the damage regions (Figure 30) obtained using the solid-shell model and PFA for the three cases are compared in these figures. The failure loads for the 1985-certification test and the 2003subcomponent test are included in Figure 29. Table 5 presents the individual load components in the lug at failure 
for the 2003-subcomponent test and W375 accident condition. The experimentally determined failure loads agree very well with the PFA predicted values, thus validating the present PFA methodology for the lug configuration. Further, all three configurations showed cleavage type failures. The failure load for the lug for the W375 accident condition $(925 \mathrm{kN})$ is greater than 1.98 times the limit load $(467 \mathrm{kN})$ [Hilgers and Winkler, 2003].

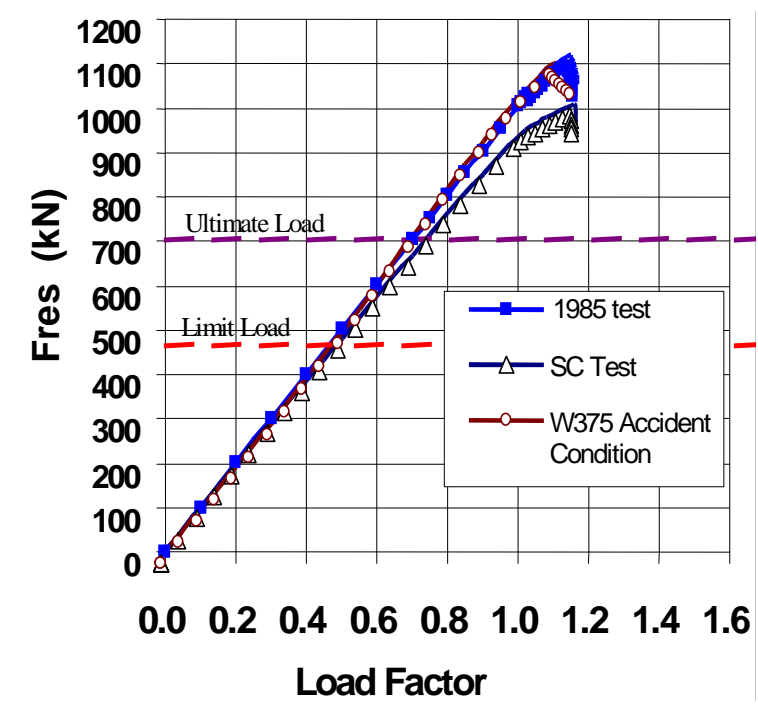

Figure 27. $F_{\text {Res }}$ vs. Load Factor Variation for 1985Certification Test, 2003-Subcomponent Test, and W375 Accident Case.

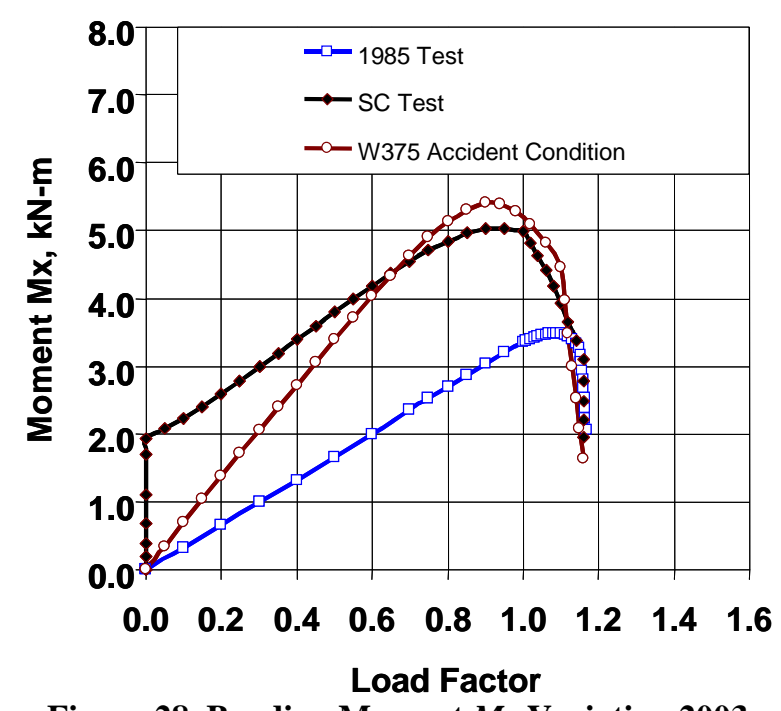

Figure 28. Bending Moment $M_{X}$ Variation 2003for 1985-Certification Test, Subcomponent Test, and W375 Accident Case.

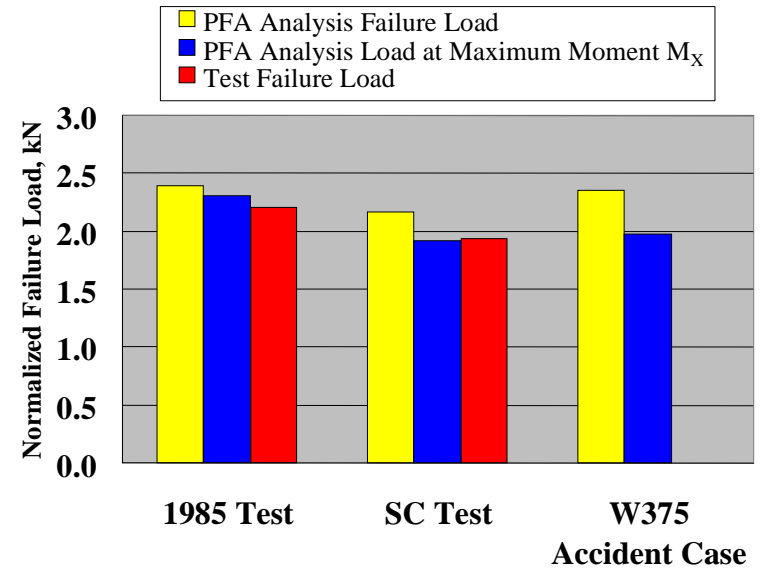

Figure 29. Failure Loads Normalized by Limit Load for 1985-Certification Test, 2003-Subcomponent Test, and W375 Accident Case.

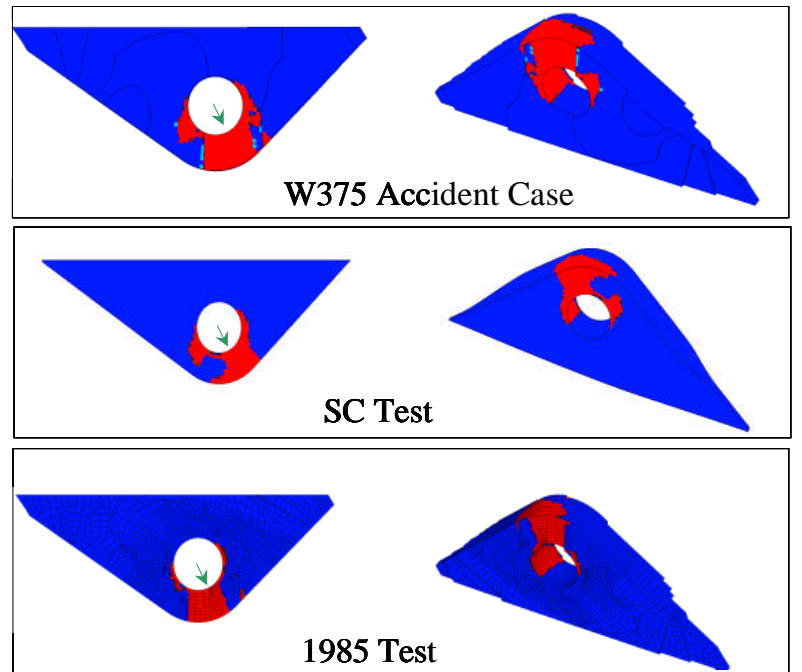

Figure 30. Comparison of Damage Predictions 1985-Certification Test, 2003-Subcomponent Test, and W375 Accident Case.

Table 5. Load Components (Normalized by Limit Load) in the Lug at Failure.

\begin{tabular}{|c|c|c|c|c|c|}
\hline Test Case & $\boldsymbol{F}_{\boldsymbol{X}}$ & $\boldsymbol{F}_{\boldsymbol{Y}}$ & $\boldsymbol{F}_{\boldsymbol{Z}}$ & $\boldsymbol{F}_{\text {Res }}$ & $\boldsymbol{M}_{\boldsymbol{X}}$ \\
\hline SC Analysis (PFA) & -374.8 & -40.39 & -812.7 & 895.9 & -5.04 \\
\hline 2003-Subcomponent Test & -381.6 & -39.10 & -822.5 & 907.0 & Not measured \\
\hline W375 Analysis (PFA) & -359.9 & -40.35 & -851.5 & 925.3 & -5.41 \\
\hline
\end{tabular}




\section{Concluding Remarks}

An analysis of the failure of the composite vertical tail of the American Airlines Flight 587 - Airbus A300-600R was performed as part of the National Transportation Safety Board's failure investigation of the accident that occurred on November 12, 2001. Two structural analysis teams, the global analysis team and the detailed lug analysis team, analyzed the vertical tail. The global analysis team evaluated the loads on each of the six lugs that attach the tail to the aluminum fuselage and determined that the right rear lug carried the largest loads compared to the design allowable. The detailed lug analysis team developed and verified user defined material and user field algorithms within the ABAQUS general-purpose finite element code. The team then performed progressive failure analyses (PFA) to predict the failure of the right rear composite lug. A global-local connection procedure was developed and validated to ensure the satisfaction of the continuity of displacements and reciprocity of tractions across the global-local interfaces and connection regions.

The right rear lug, including the neighboring fin region near the rear spar, was analyzed using two modeling approaches. In the first approach, solid-shell type modeling was used, and in the second approach, layered-shell type modeling was used. To validate the models, the solid-shell and the layered-shell modeling approaches were used in conjunction with the PFA to determine the load, mode, and location of failure in the right rear lug under loading representative of a certification test conducted by Airbus in 1985 (1985-certification test). Both analyses were in excellent agreement with each other and with the experimentally determined failure loads, failure mode, and location of failure. The solid-shell type modeling was then used to analyze a subcomponent test conducted by Airbus in 2003 as part of the failure investigation (2003-subcomponent test). Excellent agreement was observed between the PFA analyses and the experimentally determined results from the 2003-subcomponent test. Excellent agreement was also observed between the analyses of the 2003-subcomponent test and the accident condition.

From the analyses conducted and presented in this report, the following conclusions were drawn:

- The moment, $M_{X}$ (moment about the fuselage longitudinal axis) had significant effect on the failure load of the lugs. Higher absolute values of $M_{X}$ give lower failure loads. For example, an observed increase in $M_{X}$ of 45 percent from the 1985 -certification test to the 2003 -subcomponent test caused a 17 percent decrease in the failure load. Therefore, to properly test a lug under a loading condition that is representative of the flight loads, it is important to apply to the lug an accurate moment, $M_{X}$. The predicted load, mode, and location of the failure of the 1985-certification test, 2003-subcomponent test and the accident condition were in very good agreement. This similarity in results suggests that the 1985-certification and 2003-subcomponent tests represented the accident condition accurately.

- The failure mode of the right rear lug for the 1985-certification test, 2003-subcomponent test, and the accident load case was identified as a cleavage-type failure.

- For the accident case, the predicted failure load for the right rear lug from the PFA and solid-shell models was greater than 1.98 times the limit load of the lugs.

\section{References}

ABAQUS User's Manual, Vol. III, Version 6.1, Hibbitt, Karlsson \& Sorensen, Pawtucket, RI, 2000.

Averill, R.C., Non-linear Analysis of Laminated Composite Shells Using a Micromechanics-Based Progressive Damage Model, Ph.D. Thesis, Virginia Polytechnic Institute and State University, 1992.

Camanho, P.P., and Matthews, F.L., "A Progressive Damage Model for Mechanically Fastened Joints in Composite Laminates," Journal of Composite Materials, Vol. 33, No. 24, 1999, pp. 2248-2280.

Chang, F.-K., and Chang, K.Y., "A Progressive Damage Model for Laminated Composites Containing Stress Concentrations," Journal of Composite Materials, Vol. 21, 1987, pp. 834-855.

Chang, F.-K., and Lessard, L.B., "Damage Tolerance of Laminated Composites Containing an Open Hole and Subjected to Compressive Loadings: Part I-Analysis,” Journal of Composite Materials, Vol. 25, 1991, pp. 2-43.

Dávila, C.G., Ambur, D.R., and McGowan, D.M., "Analytical Prediction of Damage Growth in Notched Composite Panels Loaded in Compression,” Journal of Aircraft, Vol. 37, No. 5, 2000, pp. 898-905.

Dávila, C.G., Camanho, P.P., and de Moura, M.F.S.F., "Mixed-Mode Decohesion Elements for Analyses of Progressive

Delamination," Proceedings of the 42nd AIAA/ASME/ASCE/AHS/ASC Structures, Structural Dynamics and Materials Conference, Seattle, Washington, April 16-19, 2001(a).

Glaessgen, E.H., Pastore, C.M., Griffin, O.H., Jr. and Birger, A., "Geometrical and Finite Element Modeling of Textile Composites," Composites Part B, Vol. 27B, No. 1, 1996, pp. 43-50.

Hashin, Z., and Rotem, A., “A Fatigue Criterion for Fiber-Reinforced Materials,” Journal of Composite Materials, Vol. 7 , 1973, pp. 448-464.

Hashin, Z., "Failure Criteria for Unidirectional Fiber Composites,” J. Appl. Mech., Vol. 47, 1980, pp. 329-334.

Hilgers, R. and Winkler, E., "AAL587 Investigation Lug Sub Component Test \#1 - Results Test / FEA Comparisons," TNESGC - 1014/03, November 2003. 
MSC/NASTRAN Quick Reference Guide, Version 70, the MacNeal-Schwendler Corporation, Los Angeles, CA, 1997.

Nahas., M.N., "Survey of Failure and Post-Failure Theories of Laminated Fiber-Reinforced Composites," J. Composites Technology and Research, Vol. 8, No. 4, 1986, pp. 138-153.

Poe, C.C., Jr. and Harris, C.E., "Mechanics of Textile Composites Conference," NASA CP 3311, Parts 1 and 2, October 1995.

Rowlands, R.E., Strength (Failure) Theories and Their Experimental Correlation. In Handbook of Composites, Vol. 3. Failure Mechanics of Composites, ed. G.C. Sih and A. M. Skudra. North-Holland, 1984, pp. 71-125.

Soden, P.D., Hinton, M.J. and Kaddour, A.S., "A Comparison of the Predictive Capabilities of Current Failure Theories for Composite Laminates," Composites Science and Technology, Vol. 58, 1998(a), pp. 1225-1254.

Soden, P.D., Hinton, M.J. and Kaddour, A.S., "Lamina Properties, Lay-Up Configurations and Loading Conditions for a Range of Fibre-Reinforced Composite Laminates," Composites Science and Technology, Vol. 58, 1998(b), pp. $1011-1022$.

Young, R.D., Hilburger, M., Moore, D. and Lovejoy, A.E., "Structural Analysis for the American Airlines Flight 587 Accident Investigation - Global Analysis," Proceedings of the 46th AIAA/ASME/ASCE/AHS/ASC Structures, Structural Dynamics, and Materials Conference, AIAA-2005-2254-CP, AIAA, Austin, TX, April 18-21, 2005. 